\title{
Kinetic Measurements in Heterogeneous Catalysis
}

\section{March 2021}

Gregory Yablonsky, Guy B. Marin, Rebecca R Fushimi

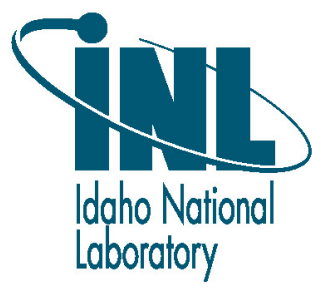




\section{DISCLAIMER}

This information was prepared as an account of work sponsored by an agency of the U.S. Government. Neither the U.S. Government nor any agency thereof, nor any of their employees, makes any warranty, expressed or implied, or assumes any legal liability or responsibility for the accuracy, completeness, or usefulness, of any information, apparatus, product, or process disclosed, or represents that its use would not infringe privately owned rights. References herein to any specific commercial product, process, or service by trade name, trade mark, manufacturer, or otherwise, does not necessarily constitute or imply its endorsement, recommendation, or favoring by the U.S. Government or any agency thereof. The views and opinions of authors expressed herein do not necessarily state or reflect those of the U.S. Government or any agency thereof. 


\title{
Kinetic Measurements in Heterogeneous Catalysis
}

\author{
Gregory Yablonsky, Guy B. Marin, Rebecca R Fushimi
}

March 2021

\begin{abstract}
Idaho National Laboratory Idaho Falls, Idaho 83415
\end{abstract}

http://www.inl.gov

Prepared for the U.S. Department of Energy Under DOE Idaho Operations Office

Contract DE-AC07-05ID14517 


\section{Kirk-Othmer Encyclopedia: Kinetics in Heterogeneous Catalysis: Measurements and Analysis}

Gregory Yablonsky, Rebecca Fushimi, Guy B. Marin

\section{Contents}

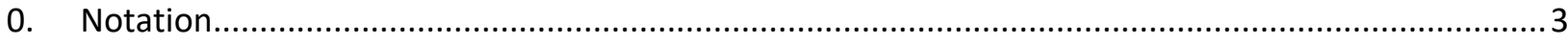

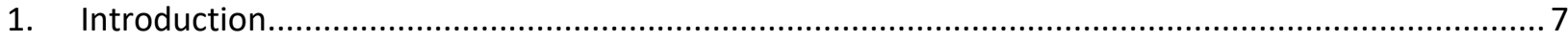

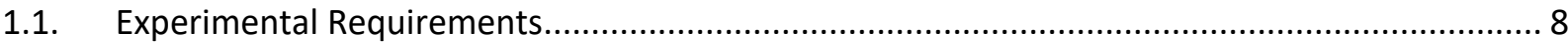

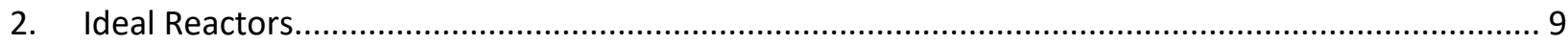

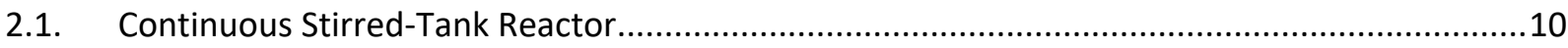

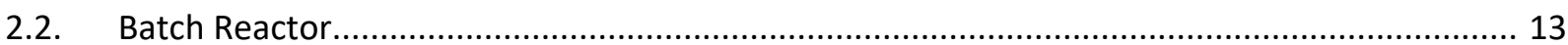

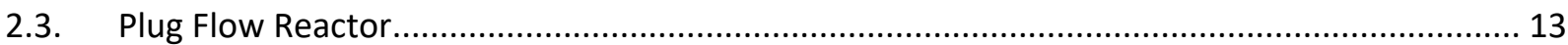

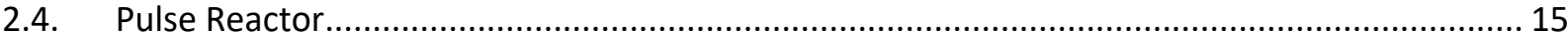

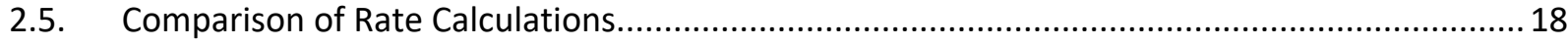

3. Nonidealities and Criteria for Kinetic Tests............................................................................ 18

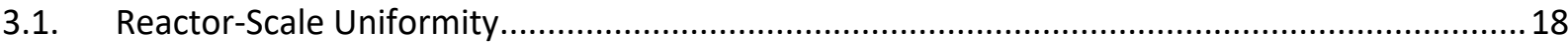

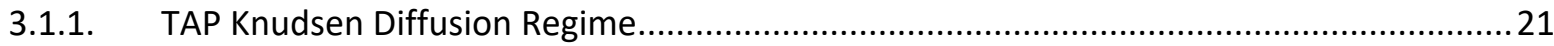

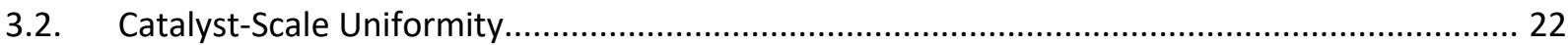

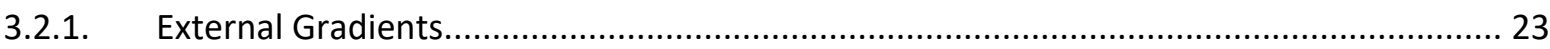

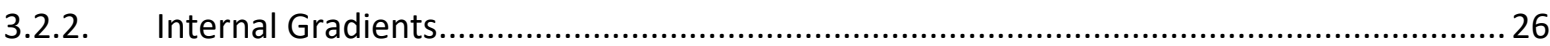

3.2.3. Example: Assessment of Transport Limitations on the Pellet Scale............................... 29

3.2.4. Experimental Methods to Assess of Transport Limitations on Pellet Scale.......................30

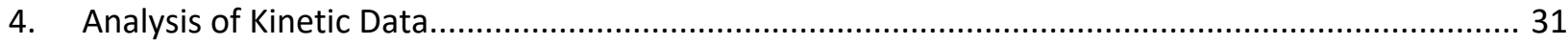

4.1. Extracting the Rate from Conversion Versus Space Time Data.............................................. 31

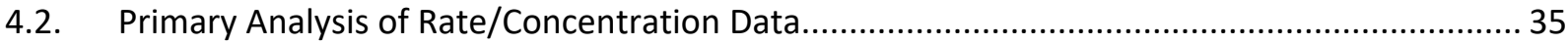

4.2.1. Stoichiometric Test: Distinguishing Single and Multiple Route Reactions.........................36

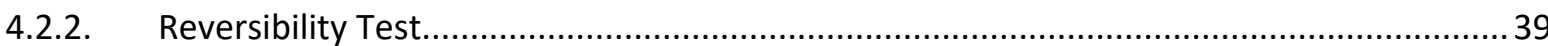

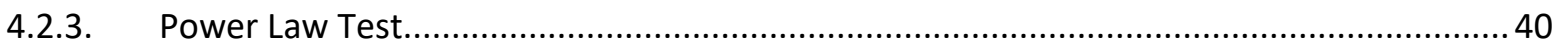

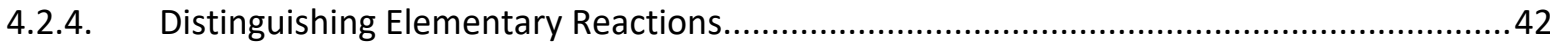

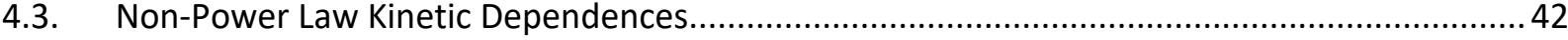




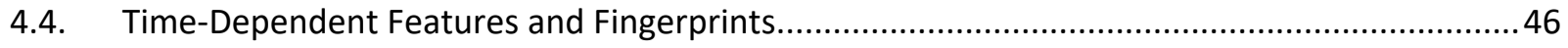

4.4.1. Concentration Time Dependence and Reaction Half-Life..............................................46

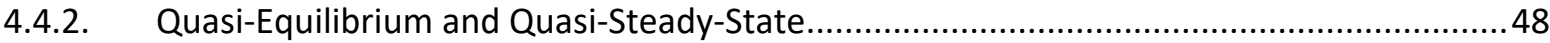

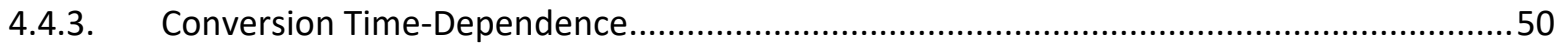

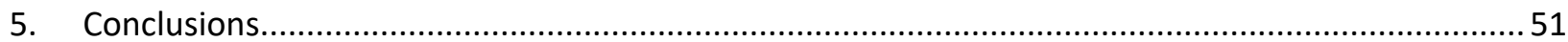

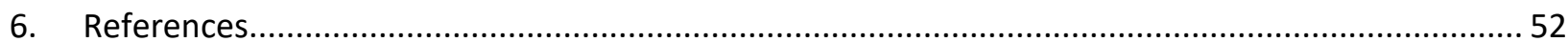




\section{Notation}

cross-sectional surface area of the reactor (available for fluid $\mathrm{m}^{2}$ flow)

specific external surface area of catalyst pellet

Maximum packed-bed dilution

concentration of component

dimensionless concentration of component

Carberry number,

Damkőhler number,

second Damkőhler number,

effective diffusivity

diffusion coefficient of component

Knudsen diffusion coefficient

molecular diffusion coefficient

diameter of channel

diameter of catalyst pellet

activation energy

molar flow rate of component

net rate of production of surface intermediate

heat transfer coefficient

Chilton-Colburn $j$-factor for mass transfer

Chilton-Colburn $j$-factor for heat transfer

equilibrium coefficient

reaction rate coefficient

Boltzmann constant $\left(1.4 \times 10^{-23} \mathrm{~J} \mathrm{~K}^{-1}\right)$

mass-transfer coefficient $\mathrm{mol} \mathrm{m}{ }^{-3}$

$\mathrm{m}^{2} \mathrm{~s}^{-1}$

$\mathrm{m}^{2} \mathrm{~s}^{-1}$

$\mathrm{m}^{2} \mathrm{~s}^{-1}$

$\mathrm{m}^{2} \mathrm{~s}^{-1}$

$\mathrm{m}$

m

$\mathrm{J} \mathrm{mol}^{-1}$

mol s ${ }^{-1}$

$\mathrm{S}^{-1}$

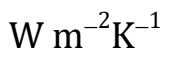

$-$

-

(mol $\left.\mathrm{m}^{-3}\right)^{\left(1_{-n}\right)} \mathrm{s}^{-1}$

$\mathrm{J} \mathrm{K}^{-1}$

$\mathrm{m} \mathrm{s}^{-1}$ 
reaction rate coefficient per unit mass of catalyst

length

m

molar mass of gas

$\mathrm{kg} \mathrm{mol}^{-1}$

number of moles of component

mol

Nusselt number

Prandtl number

heat flux

$\mathrm{W} \mathrm{m} \mathrm{m}^{-2}$

volumetric flow rate

$\mathrm{m}^{3} \mathrm{~s}^{-1}$

net rate of production of component

$\mathrm{mol} \mathrm{kg}{ }^{-1} \mathrm{~s}^{-1}$

universal gas constant

$\mathrm{J} \mathrm{mol}^{-1} \mathrm{~K}^{-1}$

radial coordinate in catalyst pellet

m

specific reaction rate (per unit mass of catalyst)

mol kg-1 $\mathrm{s}^{-1}$

Schmidt number

temperature

K

time

$\mathrm{s}$

half-life

S

superficial fluid velocity

$\mathrm{m} \mathrm{s}^{-1}$

heat-transfer coefficient

$\mathrm{W} \mathrm{m}^{-2} \mathrm{~K}^{-1}$

volume coordinate in catalyst pellet

$\mathrm{m}^{3}$

reaction volume

$\mathrm{m}^{3}$

volume of catalyst in the reactor

$\mathrm{m}^{3}$

volume of fluid in the reactor

$\mathrm{m}^{3}$

volume of catalyst pellet

$\mathrm{m}^{3}$

mean velocity

$\mathrm{m} \mathrm{s}^{-1}$

mass of catalyst in the reactor

$\mathrm{kg}$

conversion

$\mathrm{mol} \mathrm{mol}^{-1}$

axial reactor coordinate

$\mathrm{m}$ 


$\begin{array}{ll}\text { total areal density of active sites } & \mathrm{mol} \mathrm{m}^{-2} \\ \text { stoichiometric ratio } & - \\ \text { distance from equilibrium } & \mathrm{mol} \mathrm{m}^{-3} \\ \text { reaction enthalpy } & \mathrm{J} \mathrm{mol}^{-1} \\ \text { standard Gibbs energy change } & \mathrm{J} \mathrm{mol}^{-1} \\ \text { void fraction } & - \\ \text { effectiveness factor } & - \\ \text { normalized concentration of surface intermediate } & - \\ \text { mean free path } & \mathrm{m} \\ \text { thermal conductivity of catalyst pellet } & \mathrm{W} \mathrm{K}^{-1} \mathrm{~m}^{-1} \\ \text { dynamic viscosity } & \mathrm{kg} \mathrm{m}^{-1} \mathrm{~s}^{-1} \\ \text { density } & \mathrm{kg} \mathrm{m}^{-3} \\ \text { dimensionless radial coordinate in catalyst pellet } & - \\ \text { space time } & \mathrm{kg} \mathrm{s} \mathrm{mol}^{-1} \\ \text { residence time for diffusion in catalyst zone } & \mathrm{s} \\ \text { Weisz modulus } & -\end{array}$

$\begin{array}{ll}\begin{array}{cl}\text { Subscripts } \\ 0\end{array} & \text { at inlet } \\ \text { A } & \text { of component A } \\ \text { b } & \text { of catalyst bed } \\ \text { b } & \text { in bulk of fluid phase } \\ \text { cat } & \text { catalyst } \\ \text { dif } & \text { diffusion } \\ \text { eq } & \text { equilibrium } \\ \text { ex } & \text { external } \\ \mathrm{f} & \text { fluid }\end{array}$




\begin{tabular}{ll} 
in & of component \\
in, II & internal \\
of inert zone II \\
max & of surface intermediate \\
out & maximum \\
p & peak \\
p & pellet \\
s & at catalyst surface \\
ss & steady state \\
& \\
\hline Superscripts & \\
+ & forward reaction \\
\hline & reverse reaction \\
& obs
\end{tabular}




\section{Introduction}

This contribution is about the experimental determination of the rate of a heterogeneous catalytic reaction and the analysis of kinetic data. In this case, the reaction rate can be defined as the frequency at which the closed sequence of elementary steps transforming reactants into products is occurring. However, the rate of chemical reaction is not directly observed; rather, one records the rate of substance change. The rate of chemical reaction is calculated based on the rate of substance change and assumed stoichiometry of the reaction. In order to make this an intensive quantity i.e., independent of the volume in which the reaction is performed, this frequency is divided by the latter or by a quantity proportional to the latter. As the focus of what follows is on heterogeneously catalyzed reactions this can be the catalyst mass, the catalyst surface area or the total number of active sites present in the reaction volume confined by the walls of a chemical reactor. For example, the rate may be reported as one of the following:

- Net rate of consumption/production of component $i$

- Net specific rate of consumption/production of component $i$

- Rate of substance change (per unit volume of catalyst)

- Specific reaction rate (per unit mass of catalyst)

- Net rate of consumption/production of component $i$ $\mathrm{mol} \mathrm{m} \mathrm{m}^{-3} \mathrm{~s}^{-1}$

$\mathrm{mol} \mathrm{kg}{ }^{-1} \mathrm{~s}^{-1}$

$\mathrm{mol} \mathrm{m} \mathrm{m}^{-3} \mathrm{~s}^{-1}$

$\mathrm{mol} \mathrm{kg}{ }^{-1} \mathrm{~s}^{-1}$

$\mathrm{mol} \mathrm{m}^{-2} \mathrm{~s}^{-1}$

The turnover frequency (TOF) is a characteristic originally introduced by Boudart [1] is expressed as , where $R$ is the steady-state rate of reactant consumption or product generation and is the total areal density of active sites $\left[\mathrm{mol} \mathrm{m}^{-2}\right]$. The latter is typically taken from experimental chemisorption data obtained at low temperature.

The advancement of a chemical reaction leads to changes in the amounts of reactants and products which, for a stoichiometric single reaction, are connected by the stoichiometric coefficients. In many cases, for the sake of simplicity, we will assume that the investigated reaction proceeds according to a single reaction pathway, so that the net production rate of a reaction component is directly proportional to the reaction rate and that the latter equals the net production rate of any of the involved components when divided by the appropriate stoichiometric coefficient. For complex stoichiometric reactions the net production rates of the involved components are linear combinations of the reaction rates.

Different goals for kinetic measurements can be formulated:

(a) Catalyst testing, i.e., obtaining the kinetic dependences for the development and discrimination of efficient catalytic materials. Precise kinetic characterization of active materials via highthroughput screening is a part of such activity.

(b) Detailed kinetics, i.e., revealing the detailed mechanism of complex catalytic reaction via systematic kinetic studies, both steady-state and non-steady-state

(c) Industrial kinetics, i.e., obtaining data and relationships for describing and predicting the behaviour of catalytic reactors and processes at industrial scale 
(d) Finally, mathematical modeling and analysis where kinetic measurements provide data for understanding complex kinetic phenomena, e.g., oscillations, non-linear self-organization etc.

\subsection{Experimental Requirements}

To determine the net production rate, the change in chemical composition (i.e., concentration) is observed either in time or in response to operating variables such as chemical composition, flow rate or temperature. Experiments aiming to measure these net production rates can be performed in various types of reactors. Chemical reactors can be classified as either open or closed, depending on whether there is exchange of matter with the surroundings. In chemical kinetics and engineering, a closed reactor is called a batch reactor, and an open reactor is called a continuous-flow reactor.

The kinetics of reactions are measured in setups on a laboratory scale, for which the reaction conditions are easily adjustable and for which the flow pattern allows unambiguous interpretation of the measured data. In this contribution, first different types of reactors are introduced, and phenomena occurring on reactor scale are discussed. Next, reactor nonidealities that can impact kinetic measurements are dealt with; these include nonuniformity in the gas phase and on the pellet scale. From successful kinetic testing, the analysis of kinetic data for revealing the reaction mechanism is discussed. Next, the experimental data must lead to reaction rate equations which allow the design of an industrial reactor. For greater depth on this subject matter, there are numerous texts and resources that the reader may enjoy [2-9].

In practice, any chemical reaction is accompanied by physical transport of reactants and products to and from the reaction site. This also holds for the net heat produced during a reaction. So-called intrinsic chemical kinetics correspond to a situation in which the reaction rate is not affected by these mass and heat transport phenomena i.e., when the time scales at which mass and heat transfer occur are much larger than that of the chemical reaction. Where such effects may not be excluded, kinetic properties would be described as apparent.

Starting from the knowledge of the intrinsic kinetics of the reaction, industrial chemical reactors can be designed and the processes occurring in the reactor can be simulated. If the intrinsic kinetics of a reaction are unknown, then this may yield problems for scaling from lab to industrial scale. For the mutual comparison of catalysts, it is likewise important to measure intrinsic reaction rates.

The aim of this contribution is a description of the experimental techniques that enable assessment of the intrinsic kinetics of heterogeneously catalyzed reactions. Whenever possible a separation between the chemical and the physical time scales should be realized. If this is not the case, i.e., when confronted with so-called irreducible transport limitations the transport regime in the experimental reactor must be well-defined. The corresponding mathematical model then allows one to filter out the transport effects from the acquired data. For example, if Knudsen diffusion is the only transport mechanism it can be used as a "measuring stick" for extracting the intrinsic chemical kinetics.

In heterogeneous catalysis, a distinction can be made between spatial scales: the scale of the catalyst pellet corresponding to the solid phase and that of the reactor containing the pellets and the fluid surrounding them. The effects of mass and heat transfer on the reaction rate must be minimized on both spatial scales. Achieving this on the pellet scale reduces the differences in composition and temperature between the solid and the fluid phase. It allows one to ignore the latter mathematically 
and to describe the performed experiments by a so-called pseudo-homogeneous reactor model i.e., a model in which it is assumed that there is a single pseudo-phase in the reactor.

Next to ensure that the use of a pseudo-homogeneous reactor model is justified, it is also best practice to ensure that both temperature and pressure uniformity are realized and set independently from the other experimental conditions. This eliminates the need to add energy and momentum-conservation equations to the mass balances for the reactants, products and reaction intermediates, and an experimental program can be defined over a range of well-defined temperatures and total pressures. Finally, the kinetic measurement should be made in the absence of deactivating effects (e.g., sintering, coking, poisoning) and the catalyst should not change during the measurement.

To summarize, the experimental requirements of the ideal kinetic measurement include:

a) Isothermicity: Minimization of temperature gradients.

b) Uniformity of the gas and solid phase: Efficient mixing, absence of deactivation or chemical changes during the measurement. Absence of film mass and heat transfer resistances.

c) Well-defined transport: Measurement conducted in a regime controlled by the surface reaction.

d) Diversity: Conducted over a wide range of concentrations and temperatures.

In the following sections, pseudo-homogeneous reactor models are derived for some common experimental techniques and the criteria for evaluating and mitigating nonidealities will be presented.

\section{Ideal Reactors}

For any chemical component in any chemical reactor the material balance can be presented qualitatively as follows:

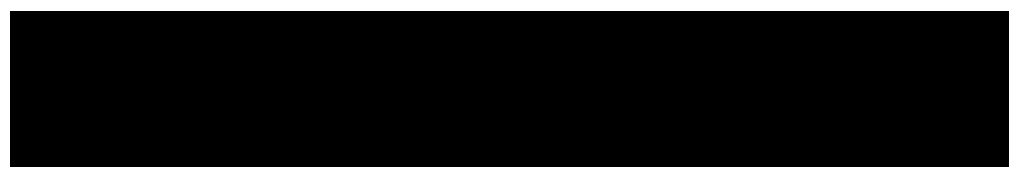

in which the temporal change of the amount of component, often termed accumulation, is its change with respect to time at a fixed position, the transport change is the change caused by motion of the component and the reaction change is the change caused by chemical reaction. Equation (1) can be used for the classification and qualitative description of different types of reactors for kinetic studies. Figure 1 shows schematic representations of a number of reactor types. There are numerous resources which can be consulted for greater detail on different types of reactors one could use [10-14]. 


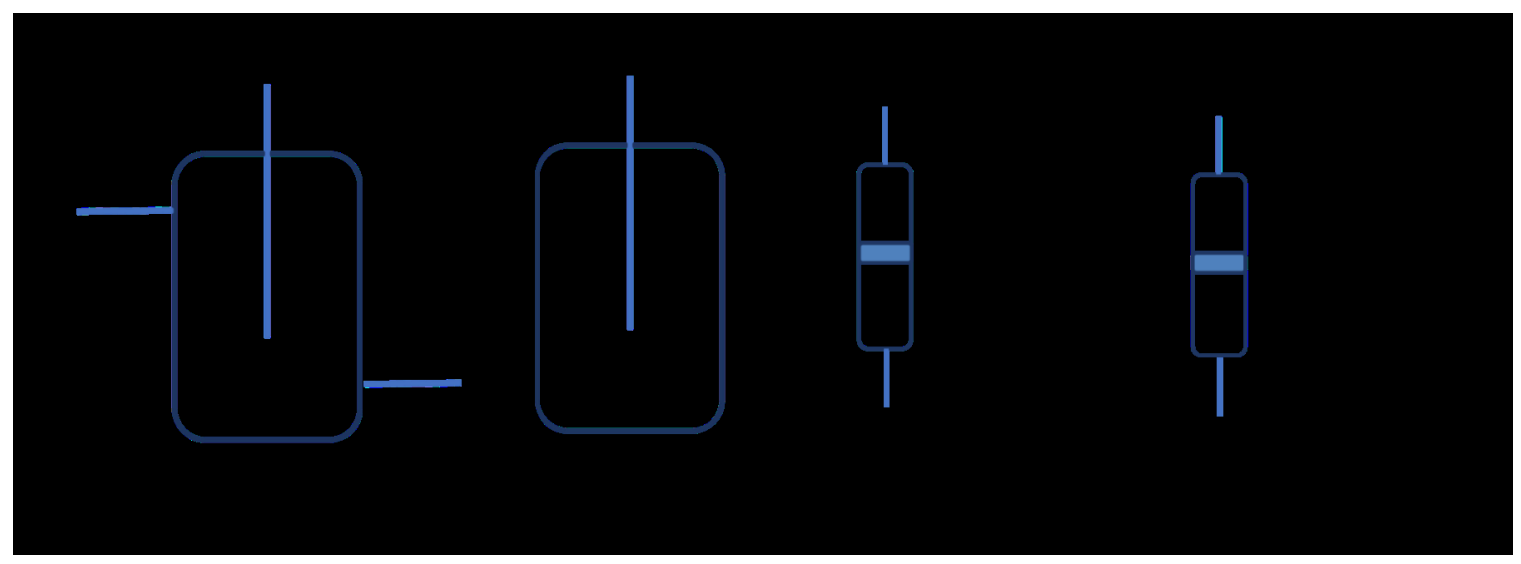

Figure 1: Reactors for kinetic experiments: a) continuous stirred-tank reactor, CSTR, b) batch reactor, c) differential plug flow reactor (PFR), d) pulse reactor.

\subsection{Continuous Stirred-Tank Reactor}

A continuous stirred-tank reactor (CSTR) is an open reactor with perfect mixing and convective inlet and outlet flows. The mass balance for a component $i$ is given by:

where $W$ is the catalyst mass $[\mathrm{kg}], n_{i}$ is the number of moles of component $i[\mathrm{~mol}]$, is the net rate of production of component $i$ per unit of catalyst mass $\left[\mathrm{mol} \mathrm{kg}{ }^{-3} \mathrm{~s}^{-1}\right], F_{i}$ is the flow rate of component $i$ $[\mathrm{mol} / \mathrm{s}]$ and $F_{\mathrm{i} 0}$ is the initial gas flow rate of component $i[\mathrm{~mol} / \mathrm{s}]$. In a steady-state CSTR the net rate of production of component $i$ can be determined from:

In chemical kinetics and chemical engineering, the concept of fractional conversion, or simply conversion, , is widely used. is dimensionless and can take values from 0 to 1 . Introducing the fractional conversion:

Equation (3) can be written as:

(5)

where is the space time $\left[\mathrm{kg} \mathrm{s} \mathrm{mol}^{-1}\right]$. The space time is proportional to the average nominal residence time in an isothermal CSTR with constant reaction volume. The denomination "space time" emphasizes that $\tau$ is also proportional to a spatial variable e.g., the volume of a continuous-flow reactor. Note that according to Equation (5) there is an unambiguous relation between the measured conversion of a component and its net rate of production for a given value of the control, i.e. the independent, variable 
space time. This does not hold for the relation between conversion of a component $i$ and the 'contact', 'holding' or 'residence' time in a reactor. The latter are not even control variables as they depend, e.g., on the stoichiometry of the reactions that are investigated.

For the simplest case, a first-order reaction $(A-B)$, the reaction rate $r\left[\mathrm{~mol} \mathrm{~kg}^{-1} \mathrm{~s}^{-1}\right.$ ] can be expressed as:

with the reaction rate coefficient $\left(\mathrm{m}^{3} \mathrm{~kg}^{-1} \mathrm{~s}^{-1}\right)$ and $C_{\mathrm{A}}$ the concentration of the component $A$. With, Equation (6) can be written as:

or:

The term, also known as the first Damköhler number, that is, the ratio of the time scale for transport from inlet to outlet of the reactor to the time scale of the reaction, is the main characteristic of the CSTR. For conversion is complete, . If ,. Knowing the conversion, an apparent first-order rate coefficient can be determined:

\section{Realizations of a CSTR}

Mixing can occur internally and/or externally. External mixing occurs by recycling a fraction of the product stream to the reaction inlet (recirculation), see Figure 2; the concentration of the circulating fluid becomes equal to that of the effluent stream. In this configuration, small deviations from perfect mixing, can introduce significant error if recycle flow rates become too low. For high recycle ratios the packed bed reactor functions can be modeled as a continuous stirred tank reactor (CSTR). Internal mixing occurs either via recycling of the reaction components or the catalyst in the reactor. Recycling of the reaction components may occur with the catalyst is fixed such as Figure 3 as well as the Berty-type reactor where the catalyst is contained in a fixed basket while an impeller rapidly recirculates the gas $[15,16]$. This configuration makes greater accuracy possible in the control of the flow rate through the catalyst bed. Alternatively, in the Carberry-type reactor, recycling of the catalyst occurs via rotation on an axis to which baskets filled with catalyst become the impeller [17] (Figure 4). Spinning the basket at high speed is effective in reducing interparticle resistances and intraparticle diffusion effects can be minimized with the use of small catalyst particles. Basket-type reactor offer good gas-solid mixing that helps to minimize thermal and concentration gradients. 


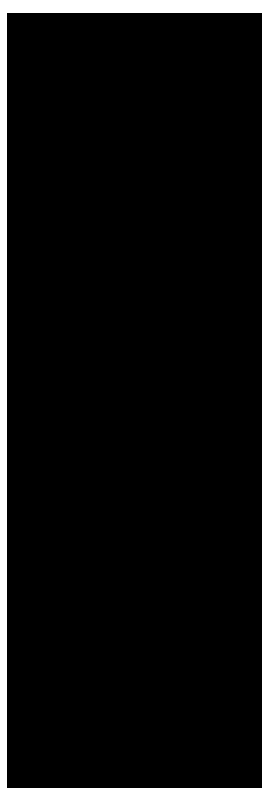

Figure 2: Schematic representation of a packed bed reactor with external recycle. Reproduced and adapted with permission from [18].

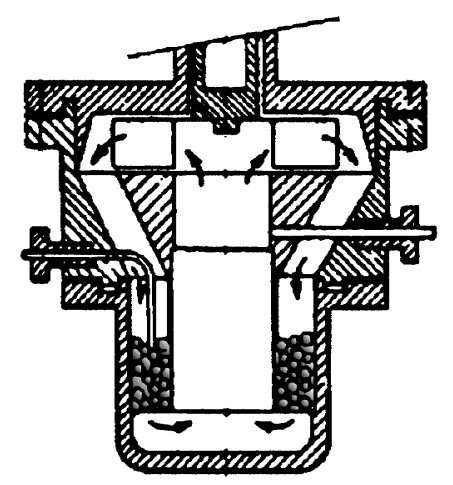

Figure 3: Schematic representation of a fixed catalyst CSTR with internal recycle. Reproduced with permission from [18].

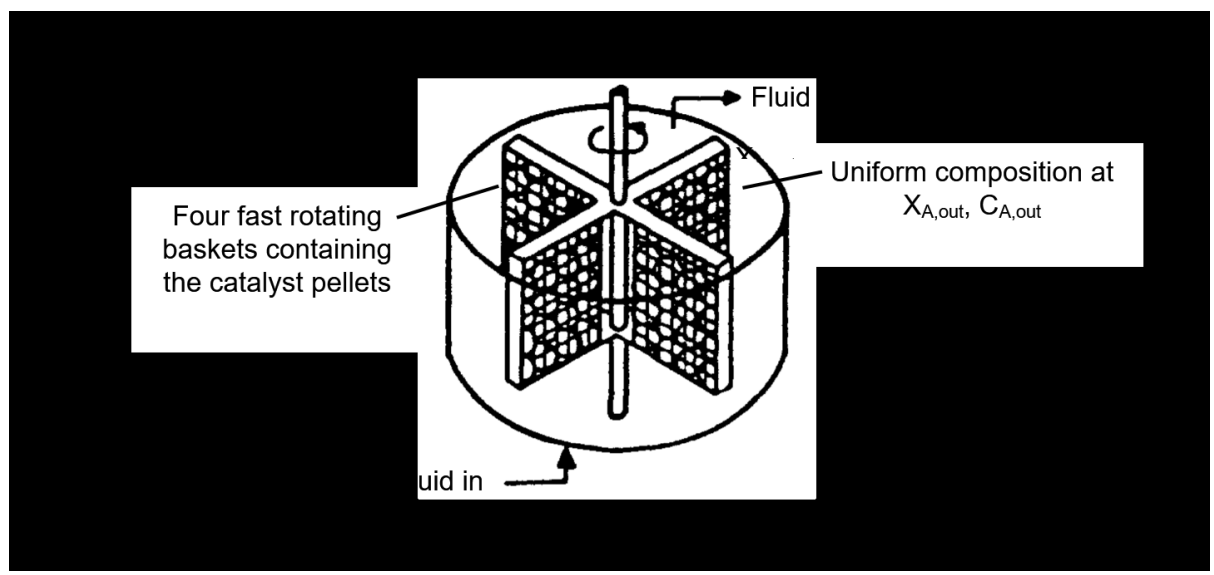

Figure 4: Schematic representation of a basket type recycle reactor. Reproduced from [19]. 
Using ideally mixed reactors to measure intrinsic reaction kinetics, has the following advantages:

- Isothermicity of the reactor is easily achieved;

- Measurements are performed for high Reynolds numbers (turbulent flow), so that concentration and temperature gradients around catalyst pellets can easily be neglected;

- The net production rate of reactants is measured directly.

Ideally mixed reactors display the follow disadvantages:

- For low operating pressure and in the case of internal mixing, it is difficult to achieve ideal mixing when using gases as reactants;

- The net production rates are not measured at inlet but at outlet conditions i.e., not easily set;

- Avoiding dead zones in the reactor is not straightforward.

\subsection{Batch Reactor}

In a batch reactor, that is, a non-steady-state closed reactor mixing can again be achieved by either internal or external recirculation. Perfect mixing results in a uniform composition throughout the reactor and, hence, simple mass balance over the whole so-called gradientless reactor. The mass balance for a component $i$ becomes:

The fractional conversion of a component in a batch reactor is defined as:

Then, Equation (10) can be written as:

i.e., as an ordinary differential equation with as an initial condition.

As the obtained experimental data typically consist of conversions the determination of a rate equation requires the integration of this differential equation. The procedure to be followed will be discussed in $\S$ 5 .

\subsection{Plug Flow Reactor}

In an ideal plug-flow reactor (PFR), Figure 5, it is assumed that perfect uniformity is achieved in the radial direction, which is the direction perpendicular to that of the flow. This is relatively easy to achieve in cylindrical tubular reactors of uniform cross-section with high aspect ratio, that is, with large length- 
to-diameter ratio. Axial diffusion effects are also neglected in the ideal case, i.e., the flow is purely convectional. It is as if a rigid plug of fluid is pushed by a piston from inlet to outlet and the streamlines followed by the individual fluid phase elements are parallel.

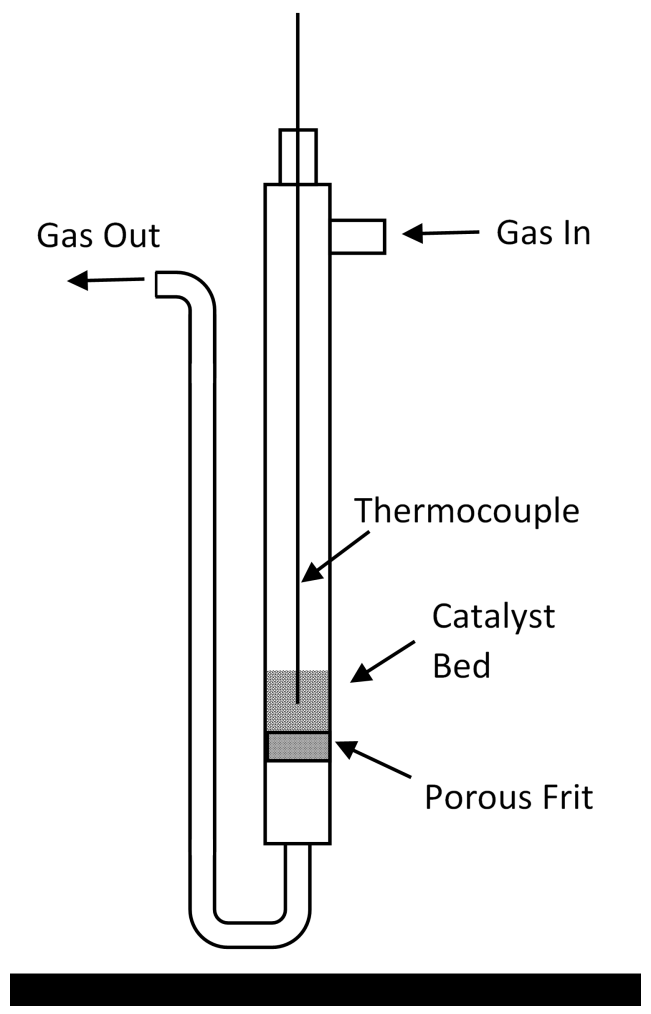

Figure 5: Schematic representation of a laboratory-scale plug flow reactor.

The composition of the fluid phase varies along the reactor, so the material balance for any component can no longer be made over the complete reactor but must be made over an infinitesimally small i.e., differential control volume:

At steady state Equation (13) can also be written as:

and the model equation for an ideal PFR is an ordinary differential equation:

with 
as an initial condition.

Note the similarity with the expression for a batch reactor, Equation (12). The only difference is the meaning of the independent variable. In the model for the batch reactor, it is the "astronomic time" during which the experiment is performed, the so-called batch time. In the model for the plug-flow reactor it is the space time.

Using a plug flow reactor has the following advantages:

- $\quad$ The setup is simple, no moving parts;

- Measurements can be performed over the entire conversion versus $W / F_{A o}$ range of interest.

The space time may be varied by changing the catalyst mass, $W$, or by changing the inlet flow rate, $F_{A 0}$. In practice the second option is chosen.

However, the plug flow reactor displays the following disadvantages:

- Isothermicity of the reactor is difficult to achieve, reactor temperature can be difficult to control, hot spots may occur with very exothermic reactions;

- Due to low flow rates, neglecting concentration and temperature gradients over the external film around the catalyst pellets is not straightforward;

- The conversion of reactants rather than their net production rate is measured.

\subsection{Pulse Reactor}

In a pulse reactor, which typically contains a fixed catalyst bed, a small amount of reactant is injected into a steady carrier gas stream. The relaxation of the outlet composition following the perturbation, provides information about the reaction kinetics. The Temporal Analysis of Products, TAP, reactor created by John Gleaves in the 1980s [20], is a special case of a pulsed reactor in which a small amount of reactant is injected into a fixed catalyst bed with the reactor exit connected to a high-throughput vacuum system [21], Figure 6. 


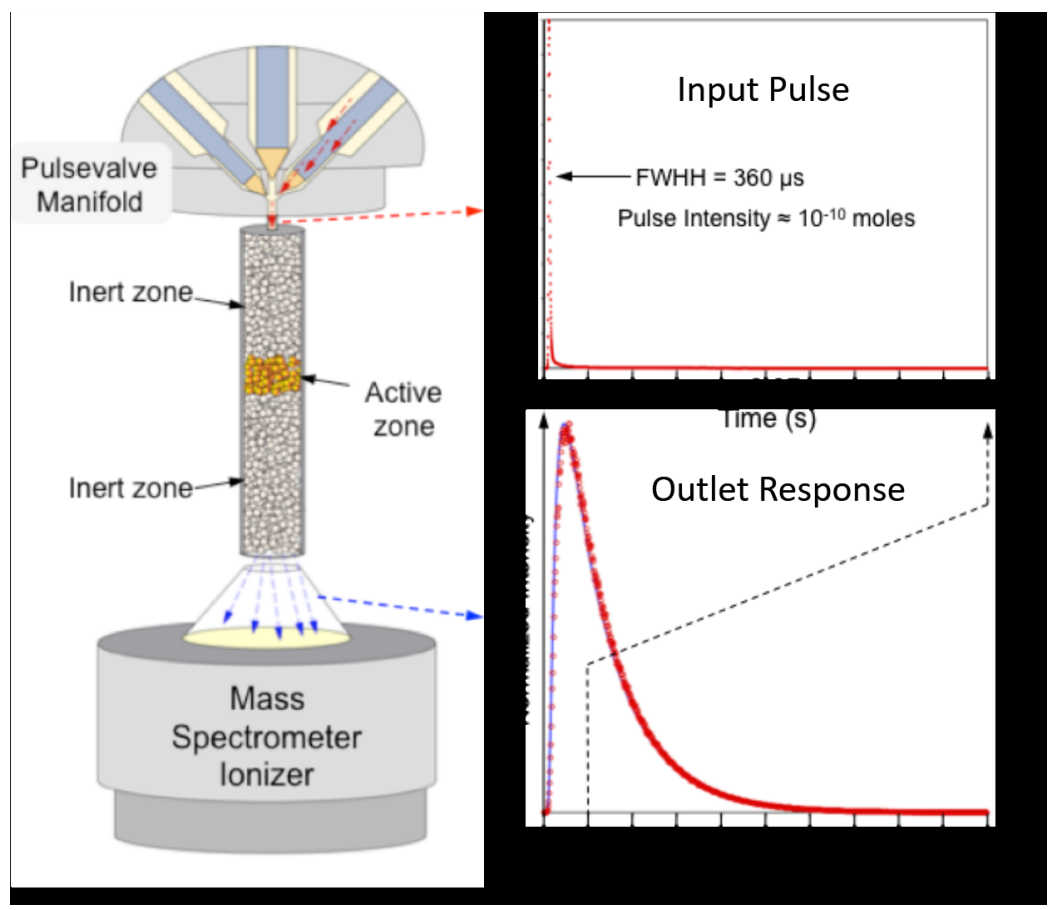

Figure 6: Schematic of a TAP reactor, illustration courtesy of Mithra Technologies, Inc..

The reactor does not use a carrier gas stream and transport only occurs by Knudsen diffusion. The small pulse size (ca. 10 nanomols) eliminates thermal effects and ensures isothermicity, even for highly exothermic or endothermic reactions. The most commonly used configuration is the thin-zone [22] where the majority of the reactor is occupied by inert particles and the catalyst comprises a thin zone at the axial center, similar to a differential plug-flow reactor. The net rate of production in the catalyst thin zone is the difference between two diffusional fluxes at either zone boundary divided by the mass of catalyst in the reactor:

However, fluxes at the catalyst zone are not directly observed and must be calculated from the reactor exit flux data, such as that depicted in Figure 7.

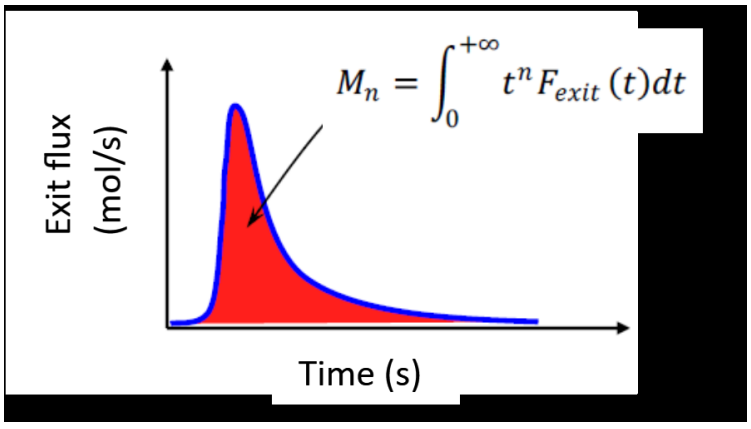

Figure 7: Exit flux is the experimental observable in the TAP reactor. 
In convection driven reactors such as the CSTR and PFR the concentration in the catalyst zone is the same as that detected at the reactor exit. However, in the TAP reactor, where diffusion is the exclusive mode of transport, the time-dependence of the flux or concentration profile changes along the reactor length. Thus, to calculate the time-dependence of the rate as in Equation (17) an inverse diffusion method must be utilized to reconstruct the fluxes at either side of the catalyst thin zone [23, 24]. Examination of the interplay of the time-dependent features of rate and concentration are what gives the technique is power however, calculations using integral quantities are more straightforward. For example, the area under the pulse response exit flux, the zeroth moment, represents the number of molecules exiting the reactor:

The reactant conversion is simply:

Higher order moments can be interpreted for their physicokinetic and the interested reader may find more details in the following references: $[25,26]$. For an irreversible reaction in a thin zone TAP reactor, the apparent rate constant can be determined from the conversion and reactor residence time according to:

where is the residence time for diffusion through the catalyst zone, , $L_{c a t}$ is the length of the catalyst thin zone, $L_{\| /}$is the length of the second symmetric inert zone $[22,27]$. Notice that Equation (20) is analogous to the expression found for the CSTR, Equation (9), which emphasizes how diffusion acts as an 'efficient impeller' to achieve perfect mixing achieved in the thin zone TAP reactor.

Use of the TAP reactor has the following advantages:

- Collection of precise non-steady-state kinetic information

- Well-defined transport;

- Perfect mixing and spatial uniformity in the catalyst when using the thin zone configuration;

- Isothermicity;

- Insignificant perturbation of the catalyst state.

However, the TAP reactor also has the following disadvantages:

- Complex setup and analysis;

- $\quad$ Low pressure conditions. 


\subsection{Comparison of Rate Calculations}

To summarize, in each device, the rate of substance change, $R$, is extracted in a different way:

- In a batch reactor $R$ is defined as a temporal derivative of concentration, ;

- In a steady-state CSTR, $R$ is an algebraic characteristics, equal to the difference between the inlet and outlet concentrations divided by the space time;

- In the steady-state PFR, $R$ is defined as the first derivative of the concentration with respect to space time or the first longitudinal derivative, ;

- For the thin zone TAP reactor, $R$ is defined as the second longitudinal derivative of concentration, .

\section{Nonidealities and Criteria for Kinetic Tests}

Each of the reactor systems described in the previous section present both advantages and disadvantages. In any reactor, isothermicity and uniformity are the critical criteria for precise kinetic measurement. A well-defined transport model can be used to determine if the measurement has been conducted in a regime controlled by the surface reaction. The common starting point is the requirement that the effects of transport phenomena should not affect the measured kinetics by more than $5 \%$, for example:

First, one must ensure uniformity of the gas and temperature on the reactor scale (e.g., radially and axially) where the catalyst is located. Next, on the scale of the catalyst pellet, one must identify the extent of gradients existing in the vicinity of the particle surface (external gradients) as well as radially from the surface towards the center of the pellet (internal gradients).

\subsection{Reactor-Scale Uniformity}

The CSTR is generally considered uniform on the reactor scale with the composition of the exit stream being the same as that in the reactor. In a plug flow reactor, there are simple design criteria that can be followed to ensure radial mixing is rapid and axial mixing is minimized. The thin zone TAP reactor and differential plug flow reactor are similar in configuration. However, like the CSTR, the TAP reactor is considered well-mixed with diffusion playing the role of 'impeller'. This has an important consequence (discussed in Section 3.1.1) for gas phase uniformity that enables kinetic measurement at high conversion in the TAP reactor (up to $80 \%$ ), whereas the plug flow reactor is restricted to $20 \%$ conversion to avoid nonuniformity.

For a plug flow reactor, Table 1 gives an overview of typical operating conditions, reactor and pellet dimensions for different process variables. These values follow from the application of criteria needed to obtain intrinsic kinetic data in the plug flow reactor. These criteria are related to deviations from the plug flow assumption and from isothermicity of the bed are discussed below. A distinction can be made 
between isothermicity in axial and in radial direction.

Table 1: Typical operating conditions and process parameters for plug flow reactors for the study of the kinetics of heterogeneously catalyzed reactions.

\begin{tabular}{|c|c|c|}
\hline Process Variable & S.I. unit & Operating Condition \\
\hline $\mathrm{P}$ & $\mathrm{Pa}$ & $2.10^{5}-10^{7}$ \\
\hline $\mathrm{T}$ & $\mathrm{K}$ & $500-1000$ \\
\hline $\mathrm{X}_{\mathrm{A}}$ & $\mathrm{mol} \mathrm{mol}^{-1}$ & $0.02-0.2$ \\
\hline \multirow[t]{2}{*}{$\mathrm{d}_{\mathrm{B}}$} & M & $10^{-2}-2.10^{-2}$ \\
\hline & $\mathrm{m}^{2} \mathrm{~m}^{-3}$ & $2.10^{2}-4.10^{2}$ \\
\hline $\mathrm{L}_{\mathrm{B}}$ & M & $10^{-1}-3.10^{-1}$ \\
\hline W & $\mathrm{Kg}$ & $10^{-4}-10^{-2}$ \\
\hline $\mathrm{U}$ & 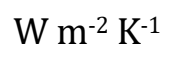 & $100-500^{*}$ \\
\hline Q & $\mathrm{W} \mathrm{m}^{-2}$ & $10^{3}-10^{6^{*}}$ \\
\hline $\mathrm{u}_{\mathrm{s}}$ & $\mathrm{m}_{\mathrm{f}}^{3} \mathrm{~m}^{-2} \mathrm{~s}^{-1}$ & $10^{-4^{* *}}-10^{2^{*}}$ \\
\hline $\mathrm{G}$ & $\mathrm{kg} \mathrm{s}^{-1}$ & $10^{-5}-10^{-3}$ \\
\hline$d_{p}$ & M & $10^{-4}-2.10^{-3}$ \\
\hline & - & $10^{-2^{* *}}-10^{4^{*}}$ \\
\hline
\end{tabular}

* only achievable via recycling, without recirculation $10^{2}$

** for gases a factor $10^{3}$ higher

Criteria for plug flow

The simplest criteria for plug flow in a packed bed are:

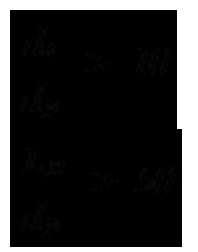

where $d_{t}$ is the diameter of the reactor $(\mathrm{m}), d_{p}$ is the diameter of the pellets $(\mathrm{m})$ and $L_{B}$ is the length of the catalyst bed $(\mathrm{m})$. Equation (22) prevents wall effects causing a radial velocity profile and hence deviations from plug flow. If Equation (23) is satisfied, then the consequence of residence time distribution may be neglected. When both criteria are met, each volume element of the flow will move through the reactor in 
the same time; as individual 'plugs'. Furthermore, there is no axial mixing between volume elements and the radial gradient of chemical concentration is negligible.

\section{Axial isothermicity}

To achieve or improve axial isothermicity of the reactor, the catalyst bed may be diluted with chemically inert material. In this way the ratio of the heat exchanging surface area to the reaction volume increases. Also reducing the tube diameter increases this ratio. If axial temperature profiles are unavoidable, then they must be accounted for by measuring the occurring profile in addition to the kinetics.

Diluting the catalyst bed with inert material may affect the conversion. A criterion for the maximal dilution degree $b_{\text {max }}\left[\mathrm{m}^{3}{ }_{\text {inert }} \mathrm{m}^{-3}{ }_{\text {inert+cat }}\right]$ is:

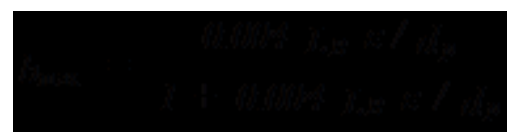

and corresponds to the requirement that the perturbing influence of the dilution on the conversion is smaller than $10 \%$ of the relative experimental error . Longer beds and a smaller pellet diameter allow greater dilution. Note that $L_{B}$ is the undiluted bed length.

\section{Radial isothermicity}

Radial isothermicity may be achieved by decreasing the tube diameter, because the resistance against radial transport decreases. However, this implies for a given pellet diameter that $d_{t} / d_{p}$ decreases, so that the plug flow criterion Equation (22) is satisfied less. To avoid this, the pellet diameter may also be reduced. This has the disadvantage of increasing the pressure drop over the reactor. In contrast to axial temperature gradients, radial temperature gradients in the bed are difficult to measure.

For a stoichiometric single reaction with kinetics:

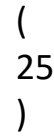

It can be shown that the reactor is radially isothermal in the sense of Equation (21) if the following criterion is satisfied:

with:

the heat transfer coefficient between bed and reactor wall [Wm $\left.\mathrm{Wm}_{\mathrm{r}} \mathrm{K}\right]$ 
the effective radial heat conductivity of the catalyst bed $\left[\mathrm{Wm}_{\mathrm{r}}^{-1} \mathrm{~K}^{-1}\right]$

1- the fraction of the reactor volume occupied by the catalyst bed $\left[\mathrm{m}_{\mathrm{B}}{ }^{3} \mathrm{~m}_{\mathrm{r}}^{-3}\right]$

1- $b$ the fraction of the catalyst bed occupied by the catalyst $\left[\mathrm{m}_{\mathrm{p}}{ }^{3} \mathrm{~m}_{\mathrm{B}}{ }^{-3}\right]$

$T_{w i}$ the internal wall temperature $[\mathrm{K}]$

The derivation of Equation (26) is given by Mears [28].

\subsubsection{TAP Knudsen Diffusion Regime}

A reliable kinetic measurement requires uniformity in both the gas phase and catalyst composition. In the plug flow reactor, the axial concentration gradient is minimized by using the differential configuration. The concentration profile can be approximated by a linear function and the reaction rate by a spatial average: $C=\left(C_{\text {in }}+C_{\text {out }}\right) / 2$. In the thin zone TAP reactor, where diffusion is the exclusive mode of transport, the rate is instead determined by the gas flux (concentration gradients) at the boundaries of the catalyst zone. In terms of conversion, nonuniformity in the TAP reactor can be estimated by:

where $L_{c}$ is the length of the catalyst zone and $L_{r}$ is the length of the reactor [29]. Figure 8, demonstrates how the gas concentration nonuniformity for both the PFR and TAP reactor changes as a function of conversion. Reliable kinetic measurement can only be made in the PFR at low conversion, $<20 \%$, however the TAP reactor can accommodate a much broader range, $c a$. $80 \%$ To better understand this result, Figure $8 \mathrm{~B}$ shows how the gas concentration changes as a function of length in the TAP reactor. At the catalyst thin zone boundaries, the difference in concentration is not as great as the difference in concentration gradients. Thus, since rate is calculated from gradients in the TAP reactor, one can collect uniform measurements even at high conversion. Greater discussion of this topic can be found in the following references: $[27,29,30]$. 

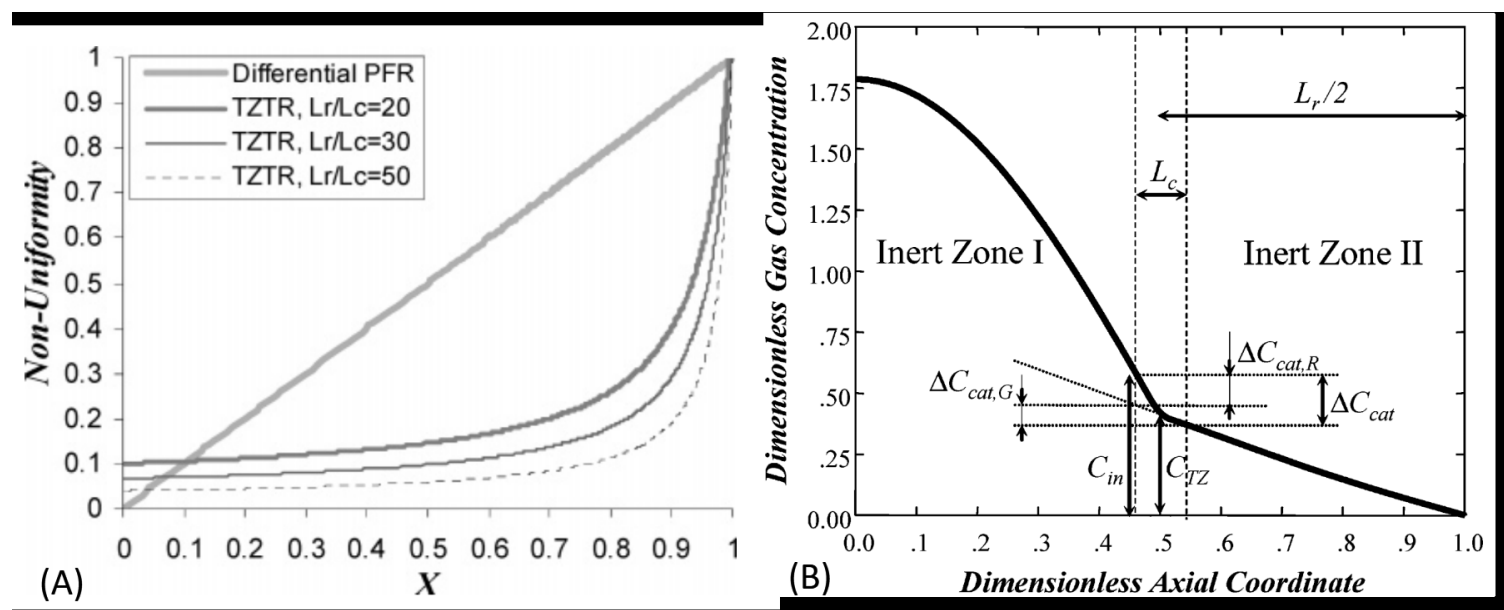

Figure 8: (A) Gas concentration nonuniformity as a function of conversion for the PFR and thin zone TAP reactor (TZTR) at different ratios of reactor length, $L_{r}$, to catalyst zone length, $L_{c}$. (B) Axial concentration profile in the thin zone TAP reactor. Dashed lines indicate non-uniformity in the catalyst zone. Reprinted (adapted) with permission from S.O. Shekhtman, G.S. Yablonsky, Thin-Zone TAP Reactor versus Differential PFR: Analysis of Concentration Nonuniformity for Gas-Solid Systems, Ind. Eng. Chem. Res., 44 ,6518-6522 (2005), American Chemical Society [29].

\subsection{Catalyst-Scale Uniformity}

In any reactor configuration, the application of a pseudo-homogeneous reactor model in analyzing experimental kinetic data is only justified if both external and internal transport limitations on pellet scale can be neglected. In other words: in order to measure intrinsic kinetics not only radial and axial temperature and concentration gradients on reactor scale must be avoided, but also gradients in and around the catalyst pellets. The latter are schematically represented in Figure 9. Only the concentrations and the temperature in the bulk of the fluid phase surrounding the pellets, $C_{b}$ and $T_{b}$, can be observed in a straightforward way. Hence, one needs to ensure that the measured production rate, $R=R(T, C)$, corresponds to the reaction occurring at $C_{s}$ and $T_{s}$. Criteria related to the pellet scale in these next sections apply to both CSTR and PFR reactors. 


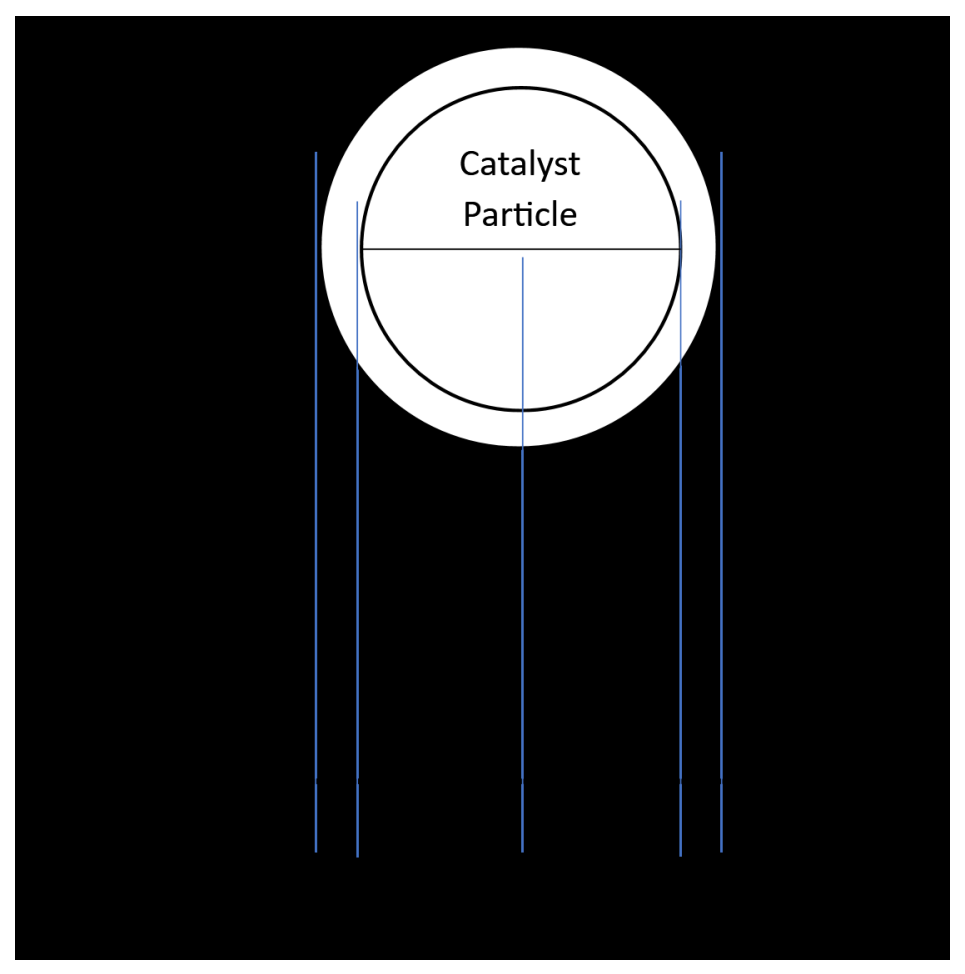

Figure 9: Possible temperature and concentration gradients in and around a catalyst pellet in a PFR and CSTR.

\subsubsection{External Gradients}

\section{External Concentration Gradients}

The effect of an external concentration gradient on the reaction rate for a first order reaction is given in the relation below:

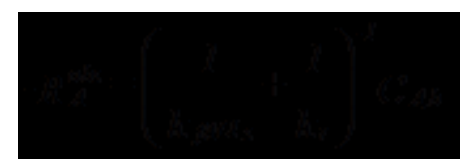

with:

$k_{f A} \quad$ mass transfer coefficient $\left[\mathrm{m}_{\mathrm{f}}^{3} \mathrm{~m}_{\mathrm{i}}^{-2} \mathrm{~s}^{-1}\right]$

$a_{s} \quad$ specific external surface area $\left[\mathrm{m}_{\mathrm{i}}{ }^{2} \mathrm{~kg}_{\mathrm{cat}}{ }^{-1}\right]$

$k_{r} \quad$ reaction rate coefficient $\left[\mathrm{m}_{\mathrm{f}}^{3} \operatorname{kg}_{\mathrm{cat}}^{-1} \mathrm{~s}^{-1}\right]$.

For reaction and transport in series at steady state:

For a partial reaction order of 1.5 , external mass transport limitations lead to deviations between the 
observed net production rate and the intrinsic production rate $R_{A}$ of less than $5 \%$ if:

After substitution of Equation (29) in Equation (30) this leads to a criterion in which only observable or calculable quantities appear:

If this is satisfied, then the measured production rate can be considered equal to the intrinsic rate $R_{A}$ which corresponds with $C_{A, b}$.

The mass transfer coefficient, $k_{f A}$, can be calculated from the $j_{D}$-factor according to Chilton-Colburn [31] when other dimensionless numbers are known:

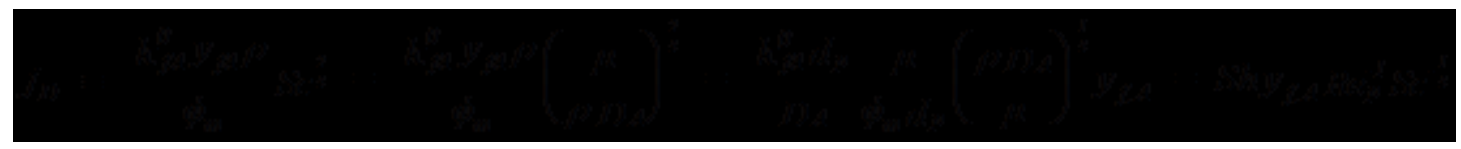

with:

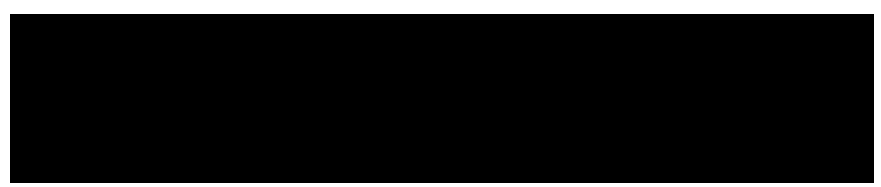

and:

$k_{f, A}^{0}$ mass transfer coefficient of $\mathrm{A}\left[\mathrm{m}_{\mathrm{f}}^{3} \mathrm{~m}_{\mathrm{i}}^{-2} \mathrm{~s}^{-1}\right]$

$\rho \quad$ density of the gas phase $\left[\mathrm{kg} \mathrm{m}_{\mathrm{f}}^{-3}\right]$

$d_{p} \quad$ pellet diameter $\left[\mathrm{m}_{\mathrm{i}}\right]$

$D_{A} \quad$ molecular diffusion coefficient $\left[\mathrm{m}_{\mathrm{f}}^{2} \mathrm{~s}^{-1}\right]$

$y_{f A} \quad$ film factor [mol fraction]

$\phi_{m} \quad$ total mass flux $\left[\mathrm{kg} \mathrm{m}_{\mathrm{r}}^{-2} \mathrm{~s}^{-1}\right]$

$\mu \quad$ dynamic viscosity $\left[\mathrm{kg} \mathrm{m}_{\mathrm{f}}^{-1} \mathrm{~s}^{-1}\right]$

The film factor $y_{f A}$ allows one to account for non-equimolar counter diffusion through the external film. For a stoichiometric single reaction it is given by: 
with:

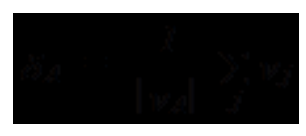

If, for this correction factor partial pressures instead of molar fractions are used, this is called the film pressure factor.

For the $j_{D}$ factor in packed beds many experimental correlations exist. An example is the correlation by Sen Gupta and Thodos [32]:

Typical values for the mass transfer coefficient are $1 \mathrm{~m}_{\mathrm{f}}^{3} \mathrm{~m}_{\mathrm{i}}^{-2} \mathrm{~s}^{-1}$ for transfer from the gas phase and $10^{-3}$ $\mathrm{m}_{\mathrm{f}}^{3} \mathrm{~m}_{\mathrm{i}}^{-2} \mathrm{~s}^{-1}$ for transfer from the liquid phase.

\section{External Temperature Gradients}

For heat transport in series with thermal energy developed by the reaction, the following holds a steady state:

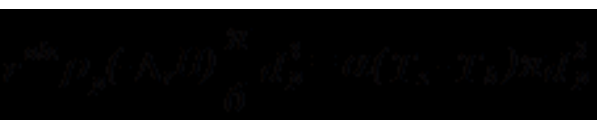

The heat transfer coefficient can be calculated from the $j_{H^{-}}$factor according to Chilton-Colburn:

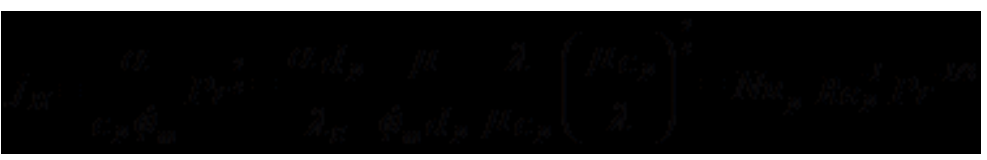

with:

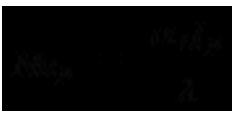

For the $j_{H}$ factor in packed beds, experimental correlations exist, e.g., the correlation of de Acetis and Thodos [33]: 


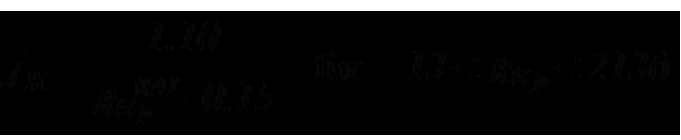

(40)

Typical values for the heat transfer coefficient are between $10^{2}-10^{3} \mathrm{~W} \mathrm{~m}_{\mathrm{i}}^{-2} \mathrm{~K}^{-1}$ for transfer from the gas phase.

The criterion for the negligibility of external temperature gradients is:

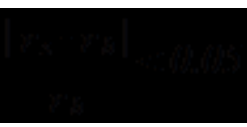

(41)

For a reaction rate equation of the form:

It follows via Taylor expansion of $r_{w}$ about $T_{b}$ :

If exclusively external temperature gradients occur, then neglecting the higher order terms in the Taylor series yields the following relation between the reaction rate at bulk temperature and that at the external surface holds:

Note that:

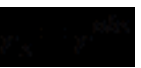

Substitution in Equation (41) yields as criterion:

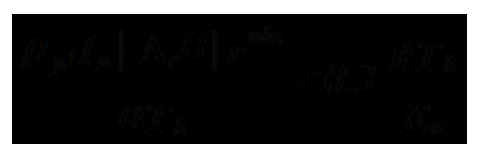

in which exclusively observable or computable quantities appear.

\subsubsection{Internal Gradients}

Internal Concentration Gradients 
The effectiveness factor of a reaction volume is defined as:

Pore diffusion limitations are negligible if the effectiveness factor is sufficiently high:

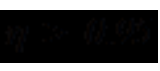

Introducing the Weisz modulus, similar to the observable Thiele modulus, which is based on the observed rate, $r^{\text {obs }}$, i.e., in the possible presence of pore diffusion limitations:

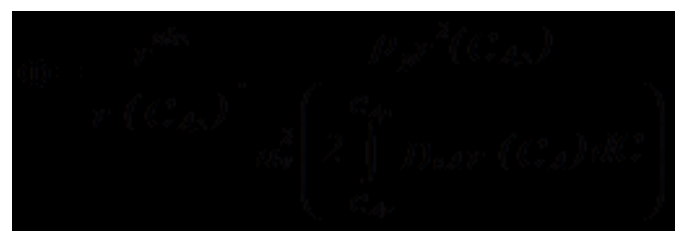

(50)

with an effective diffusion coefficient:

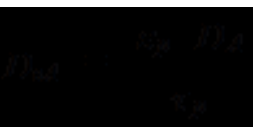

accounting for the porosity, $\varepsilon_{p}$, and the tortuosity, $\tau_{p}$, of the catalyst pellets and which for $n^{\text {th }}$ order kinetics becomes:

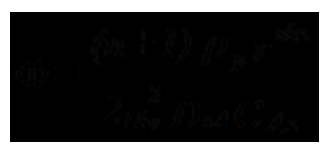

leads to a criterion for the negligibility of internal diffusion limitations:

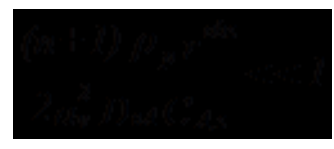

in which only observable or computable quantities appear.

\section{Internal Temperature Gradients}

Analogous to equation (44) the net production rate of a component $A$ in a catalyst pellet may be written as a Taylor series approximation around $T_{s}$ : 
At the external surface of the pellet holds $T=T_{s}$ and in the center of the pellet the temperature gradient is zero due to symmetry, hence, $T$ reaches an extremum there. The simplest internal temperature profile satisfying these conditions is given by a parabolic expression:

? is a positive constant for an exothermic reaction. Substituting Equation (55) in Equation (54) and integration the pellet volume yields, after multiplication with $\rho_{p}$, the extensive production rate:

or:

The average specific production rate, which is nothing more than the observed specific production rate, follows from:

So that the following relation holds:

If cannot deviate more than $5 \%$ from $R_{w, s}$, then the criterion for the negligibility of internal temperature gradients becomes:

? may be eliminated from (60) via derivation of an energy balance over the external surface of the catalyst pellet at the steady state. For a single reaction:

with $S_{p}$ the external pellet surface area in $\left[\mathrm{m}_{\mathrm{p}}{ }^{2}\right]$ and $V_{p}$ the pellet volume in $\left[\mathrm{m}_{\mathrm{p}}{ }^{3}\right]$.

Via substitution of (55) and because $\quad$ for spherical geometry, (61) becomes:

Solution of (62) for ? and substitution in (60) yields as criterion: 


\subsubsection{Example: Assessment of Transport Limitations on the Pellet Scale}

The follow example is adapted after Doraiswamy et al. [34] $n$-butenes may be isomerized to iso-butene over a fluorinated alumina catalyst at $673 \mathrm{~K}$. The reaction rate was measured in a reactor with ideal mixing. A typical measured reaction rate is: $r_{v}=5.25 \mathrm{~mol} \mathrm{~m}^{-3} \mathrm{~s}^{-1}$. To verify if the reaction rate was measured without pellet scale transport limitations, it is sufficient to apply the criteria discussed in this chapter. Table 2 summarizes the required data.

Table 2: Required values to assess transport limitations for butane isomerization

\begin{tabular}{|l|l|l|l|}
\hline Pellet density & $?_{p}$ & 1500 & $\mathrm{~kg}_{\text {cat }} \mathrm{m}_{\mathrm{p}}^{-3}$ \\
\hline Specific external surface area & $a_{s}$ & 8.78 & $\mathrm{~m}_{\mathrm{i}}{ }^{2} \mathrm{~kg}_{\mathrm{cat}}{ }^{-1}$ \\
\hline Pellet diameter & $d_{p}$ & $3.4 \times 10^{-4}$ & $\mathrm{~m}_{\mathrm{p}}$ \\
\hline Superficial mass flow rate & $?_{\mathrm{m}}$ & 7.31 & $\mathrm{~kg} \mathrm{~m}_{\mathrm{r}}^{-2} \mathrm{~s}^{-1}$ \\
\hline Gas density & $?$ & 1.014 & $\mathrm{~kg} \mathrm{~m}_{\mathrm{f}}{ }^{3}$ \\
\hline Gas viscosity & $?$ & $1.75 \times 10^{-5}$ & $\mathrm{~kg} \mathrm{~m}_{\mathrm{f}}^{-1} \mathrm{~s}^{-1}$ \\
\hline Gas heat capacity & $C_{p}$ & $2.717 \times 10^{3}$ & $\mathrm{~J} \mathrm{~kg}^{-1} \mathrm{~K}^{-1}$ \\
\hline Eff. diffusion coefficient & $D_{e A}$ & $3.610^{-7}$ & $\mathrm{~m}_{\mathrm{f}}^{3} \mathrm{~m}^{-1} \mathrm{~s}^{-1}$ \\
\hline Mol. diffusion coefficient & $D_{A}$ & $2.110^{-5}$ & $\mathrm{~m}_{\mathrm{f}}^{3} \mathrm{~m}^{-1} \mathrm{~s}^{-1}$ \\
\hline $\begin{array}{l}\text { Gas phase heat conduction } \\
\text { coefficient }\end{array}$ & $?$ & $8.06 \times 10^{-2}$ & $\mathrm{~W} \mathrm{~m}_{\mathrm{f}}^{-1} \mathrm{~K}^{-1}$ \\
\hline Pellet heat conduction coefficient & $?_{p}$ & 1.0 & \\
\hline$n$-butene bulk concentration & $C$ & 13.7 & $\mathrm{~W} \mathrm{~m}_{\mathrm{f}}^{-1} \mathrm{~K}^{-1}$ \\
\hline Reaction enthalpy & $?_{r} \mathrm{H}$ & $5.852 \times 10^{3}$ & $\mathrm{~mol} \mathrm{~m}_{\mathrm{f}}^{-3}$ \\
\hline Activation energy & $E_{a}$ & $3.49 \times 10^{4}$ & $\mathrm{~J} \mathrm{~mol}^{-1}$ \\
\hline Bulk temperature & $T_{b}$ & 673 & $\mathrm{~K}$ \\
\hline
\end{tabular}

Calculate the dimensionless groups:

Calculate $k_{f, A}$ and $\alpha$ from $j_{D}$ and $j_{H}$ : 
External mass transport:

According to (31) it must hold that:

(71)

Substitution of the values from Table 2 yields:

Hence, no external mass transport limitations exist.

External heat transport:

Introduction of criterion (47):

Hence, the effects due to external heat transport are negligible.

Internal mass transport:

Calculate the Weisz modulus according to equation (50):

Note that first order kinetics and a spherical shape were assumed. For higher order kinetics (51) is still easily satisfied. Hence, internal mass transport effects are negligible.

Internal heat transport:

Introduction of the data in criterion (63):

Indicates that temperature gradients in the pellets do not occur.

\subsubsection{Experimental Methods to Assess of Transport Limitations on Pellet Scale}

To conclude this section, a number of practical tests are given to assess the occurrence of transport limitations. 


\section{Test for the occurrence of external concentration gradients}

One varies the molar flow rate, $F_{A, 0}$, but keeps the space time, $W / F_{A, 0}$, constant (see Figure 10 ). From equations (15), (16) and (26) for a first order reaction and transport in series follows for the conversion in an ideal plug flow reactor:
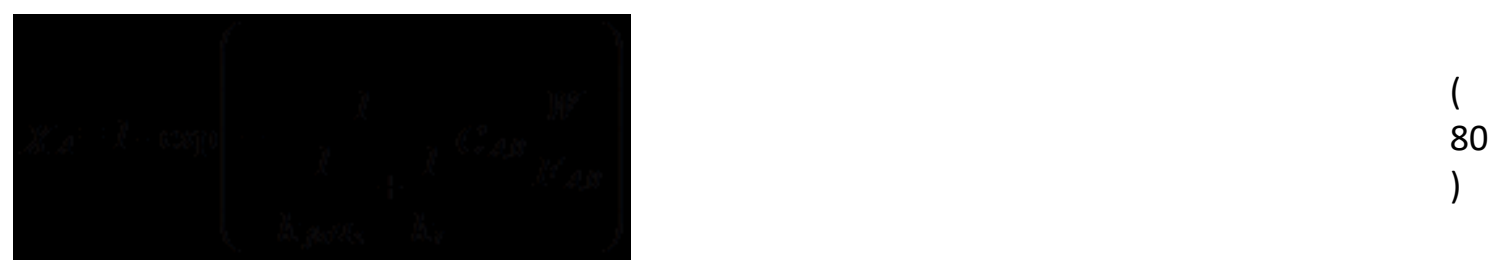

Because the mass transfer coefficient is dependent on the superficial gas velocity and, hence, on the molar flow rate, $X$ will vary as a function of $F_{A, O}$ for external mass transport limitations.
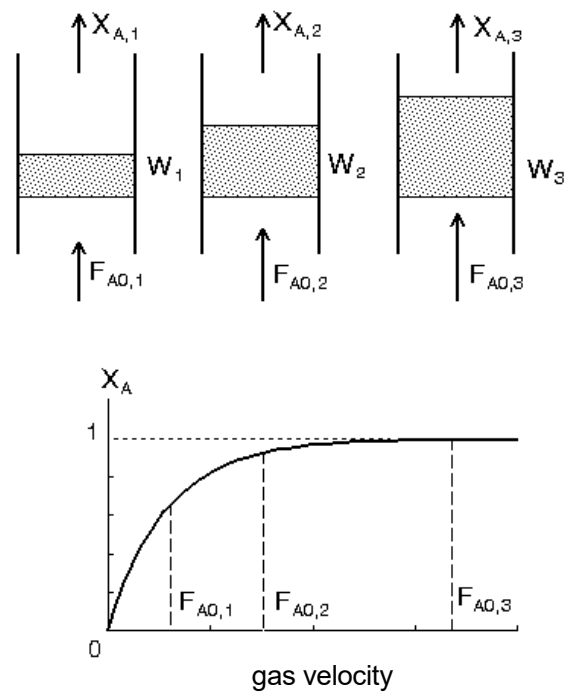

Figure 10: Conversion versus gas velocity for constant space time.

\section{Test for the occurrence of internal concentration gradients}

A way to test whether internal transport limitations occur, is to measure the reaction rate for identical reaction conditions, but for different pellet diameters. As long as there is no internal concentration profile, the effectiveness factor is equal to unity and the reaction rate is independent of the pellet diameter. From a critical pellet diameter this no longer holds, and the observed rate decreases with increasing pellet diameter.

\section{Analysis of Kinetic Data}

\subsection{Extracting the Rate from Conversion Versus Space Time Data}

Once sufficient data is available over a range of conditions (i.e., of temperatures, pressures, feed composition and conversion) kinetic modeling may begin. This implies the description of the obtained 
data using reaction rate equations. The data are often obtained as shown in Figure 11, i.e., in the form of a number of so-called $X_{A}$ vs. $W / F_{A O}$ curves. This diagram is related to first-order kinetics. Each of these curves corresponds to a set of operating conditions and results typically from measuring the conversion of $A$ at different inlet flow rates for a given mass of catalyst.

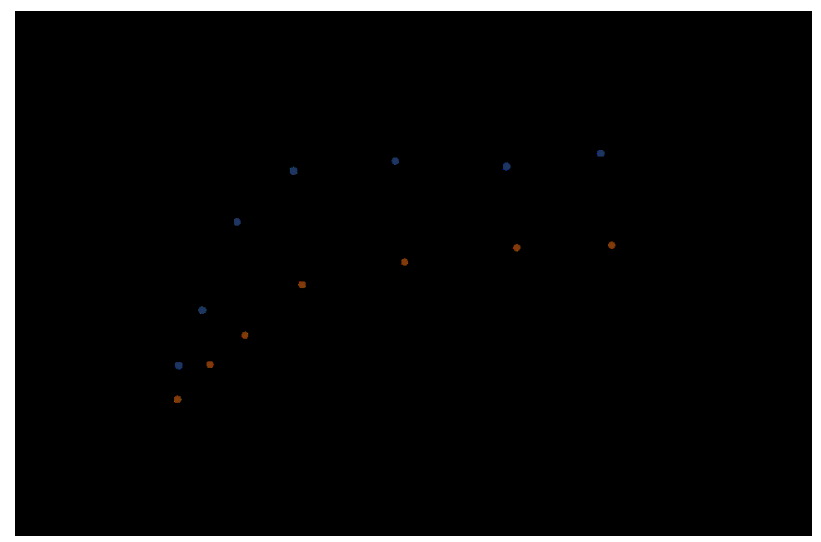

Figure 11: Illustration of experimental data collected in a plug flow reactor, conversion of $A, X_{A}$, versus space time, $W / F_{A 0}$.

As mentioned previously, the uniformity of the chemical composition in the reactive zone is one of the main requirements for kinetic measurements. In the CSTR and differential plug flow reactor, this uniformity is achieved only at low conversion, generally less than $20 \%$. The low conversion domain, or the domain of differential operation is indicated by the dashed line in Figure 11, where a linear relation between the conversion and space time is observed. Above the dashed line, the domain of integral operation exists. This region is related to kinetic measurements at higher conversions in the plug flow reactor (PRF), in this case referred to as an integral reactor. Extracting intrinsic kinetic data is based on differential operation. Typically, integral reactor operation is used for performance evaluation nearer to industrial operating conditions as well as for catalyst deactivation or ageing studies.

In the PFR, the experimental reaction rate follows from Equation (15), ; it is a slope of the tangent to the $X_{A}$ versus $W / F_{A O}$ curve for a given $W / F_{A O}$ at a corresponding composition. After completing the experimental program, a number of measurements of reaction rates will be available for a set of conditions, $i$, of temperature, $T_{i}$, and concentrations, $C_{i}$ :

These data must now be regressed using a reaction rate equation, i.e., a mathematical expression theoretically relating the reaction conditions and the reaction rate: 
The constant quantities appearing in Equation (82) are the so-called kinetic parameters. The values of these parameters are usually insufficiently known with accuracy needed to allow a reliable reactor design. Hence, an estimation of these values using the available experimental data, Equation (81) is necessary. This is done by application of the least squares criterion, i.e., the minimization of:

by adjusting the values of the kinetic parameters i.e., a so-called parameter estimation.

The estimation of kinetic parameters based on experimental data obtained according to Equation (15) is called the differential method of kinetic analysis of plug flow reactor data. For an integral plug flow reactor this method requires the differentiation of $X_{A}$ vs. $W / F_{A 0}$ curves, which can be a source of error due to experimental noise. In that case the so-called integral method of data processing is recommended. The latter requires a parameter estimation by minimization of an objective function based on the observed conversions:

The calculated conversions, are obtained via integration of the continuity equation for $A$ Equation (15) after substitution of Equation (82):

for each of the experimental conditions $i$.

\section{Example}

Let us illustrate the differential method of kinetic analysis for data obtained in a plug flow reactor. In what follows it will be assumed that the experiments have been performed at conditions such that intrinsic kinetic data have been obtained i.e., that the criteria discussed in Section 3 are met. This allows the use of the pseudo-homogeneous reaction models derived in Section 2. Here the independent variable is the space time which is varied by a change in the flow rate. The reaction rate in a plug flow reactor follows from the space time derivative. In the partial oxidation reaction of methane over platinum, the reactant conversion data in Table 3 (plotted in Figure 12) was recorded as a function of space time. Low conversions were achieved by high total flow rates and low reactant partial pressures with methane in excess.

Table 3: Example data from methane partial oxidation in a differential plug flow reactor.

\begin{tabular}{|l|l|l|l|}
\hline $\begin{array}{l}\text { Space Time, } \\
\text { [g mol }^{-1} \text { ] }\end{array}$ & $\begin{array}{l}\text { Methane } \\
\text { Conversion }\end{array}$ & $\begin{array}{l}\text { Oxygen } \\
\text { Conversion }\end{array}$ & CO Selectivity \\
\hline 10.5 & 0.013 & 0.062 & 0.36 \\
\hline 12.5 & 0.015 & 0.073 & 0.33 \\
\hline
\end{tabular}




\begin{tabular}{|l|l|l|l|}
\hline 14 & 0.017 & 0.081 & 0.32 \\
\hline 16.5 & 0.019 & 0.101 & 0.30 \\
\hline 20 & 0.024 & 0.117 & 0.28 \\
\hline 25 & 0.028 & 0.145 & 0.24 \\
\hline 33 & 0.039 & 0.172 & 0.20 \\
\hline 50 & 0.065 & 0.275 & 0.15 \\
\hline
\end{tabular}

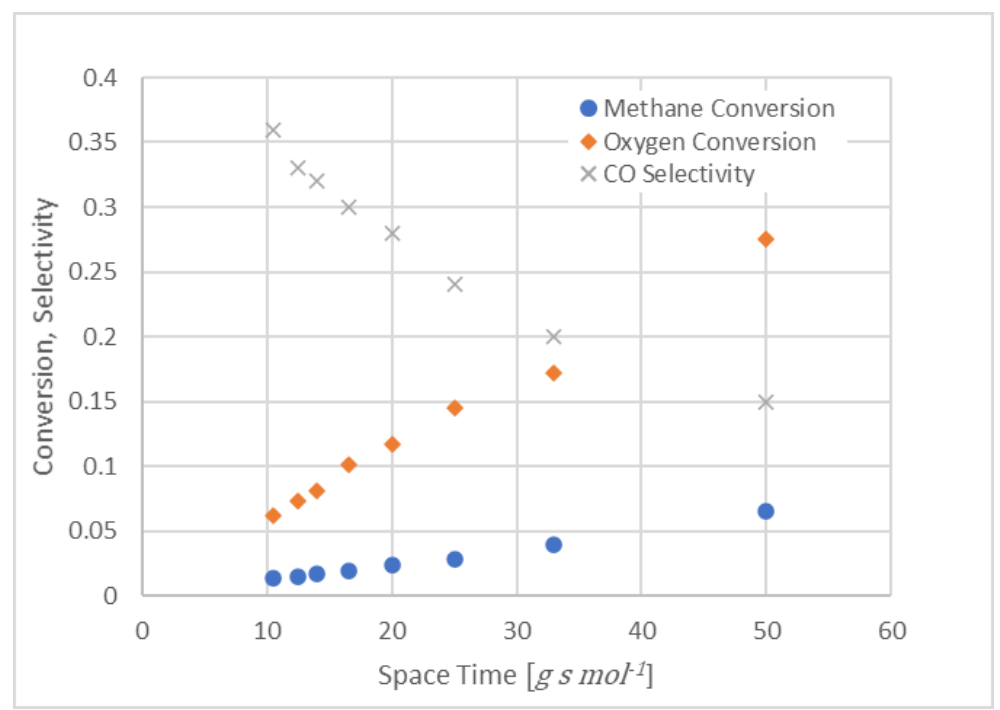

Figure 12: Example data from methane partial oxidation in a differential plug flow reactor.

The derivative in Equation (15) can be approximated by the difference. In the first interval of space time, $\left[10.5 \mathrm{~g} \mathrm{~s} \mathrm{~mol}^{-1} ; 12.5 \mathrm{~g} \mathrm{~s} \mathrm{~mol}^{-1}\right]$ the rate of methane conversion is:

Likewise, for oxygen:

One drawback to the differential method of data analysis is that even relatively mild experimental noise will lead to unsatisfactory stability in the rate calculation; see rate data plotted in Figure 13. If reactor operation is in the differential regime (i.e. depending on the partial reaction orders, $<20 \%$ conversion) the rate can be calculated using the CSTR Equation (5), . For example, at the first space time data point the rate of methane conversion is:

similarly, the rate of oxygen conversion is: 
To calculate the rate of product formation, product yield should be used in place of reactant conversion. In this example, oxygen is the limiting reactant and hence oxygen conversion can be used to calculate $\mathrm{CO}$ yield from the selectivity measurement. The rate of $\mathrm{CO}$ formation at the first data point is thus:

The rate calculations for the reactants are included in Figure 13 using both Equations. With a large enough sample of data points, the kinetic parameters can be estimated using a minimization method as described in Equation (84) where and the conversion data are calculated from Equation (85) over the entire range of observed conversions. Of course, more sophisticated methods of parameter estimation, curve fitting and data filtering exist but each comes with its own set of assumptions and biases that should be considered. Note that this analysis has to be preceded by a verification if the intrinsic nature of the obtained data i.e., a verification of the absence of transport limitations on both catalyst particle and reactor scale.

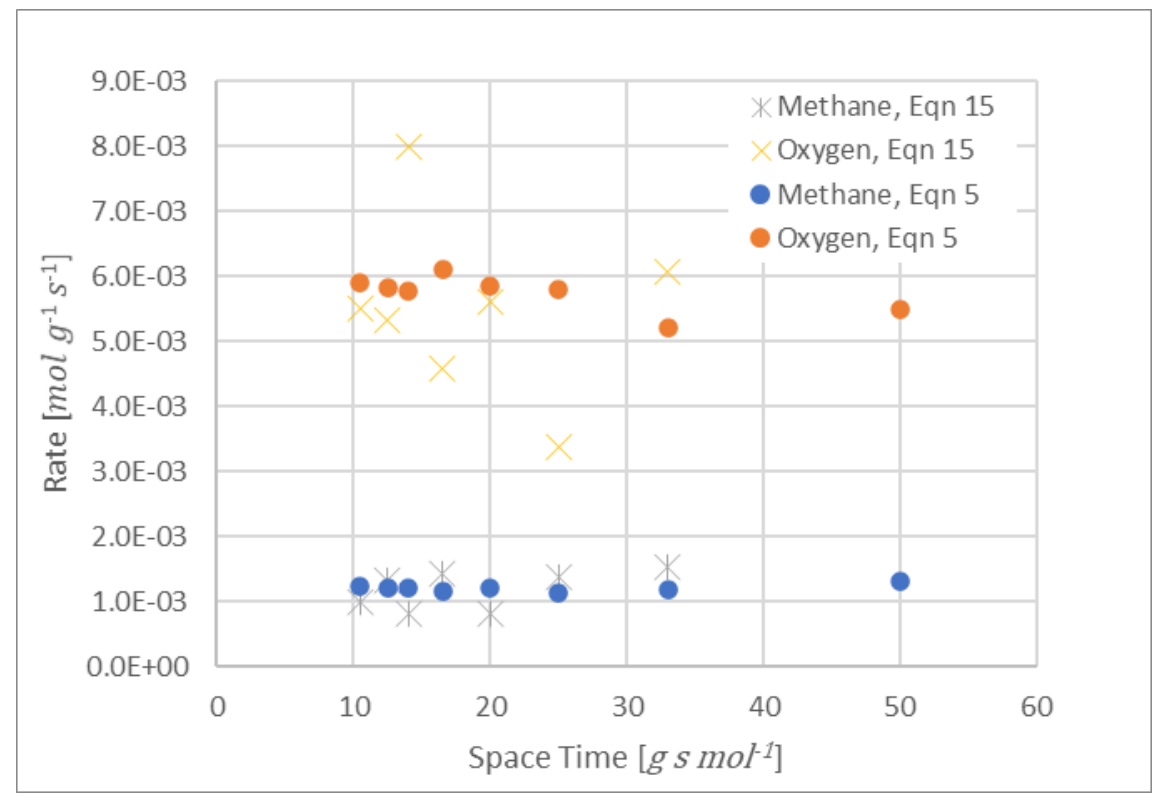

Figure 13: Rate data for methane partial oxidation in a plug flow reactor calculated using Equation (15) and Equation (5).

\subsection{Primary Analysis of Rate/Concentration Data}

Once conversion vs. space time data are converted to rate vs. concentration data as described in the previous section, a reaction rate equation may be regressed for reactor design and performance evaluation. There are different types of rate equations, Equation (82), that may be used to express the reaction rate in terms of reaction conditions. In this work we will make the distinction between power law equations such as , , , and Langmuir-Hinshelwood-Hougen-Watson (LHHW) equations such as and; where $C_{r}$ and $C_{p}$ are concentration of reactants and products, respectively, and $C_{i}$ are concentrations of the $i^{\text {th }}$ substance. A number of primary tests should be first applied to the rate/concentration data to reveal useful features of the reaction mechanism and determine the appropriate type of reaction rate expression that should be used:

a) Does the reaction occur via a single route or multiple routes? 
b) Is the reaction irreversible or reversible?

c) Can a power law expression be used?

d) Are the reactions complex or elementary?

This series of test, summarized in Table 4, will reveal important primary assumptions for the kinetic model and lead to the appropriate mathematical description. The following sections will work through the criteria for each kinetic test.

Table 4: Primary analysis mapping of reaction rate kinetic data.

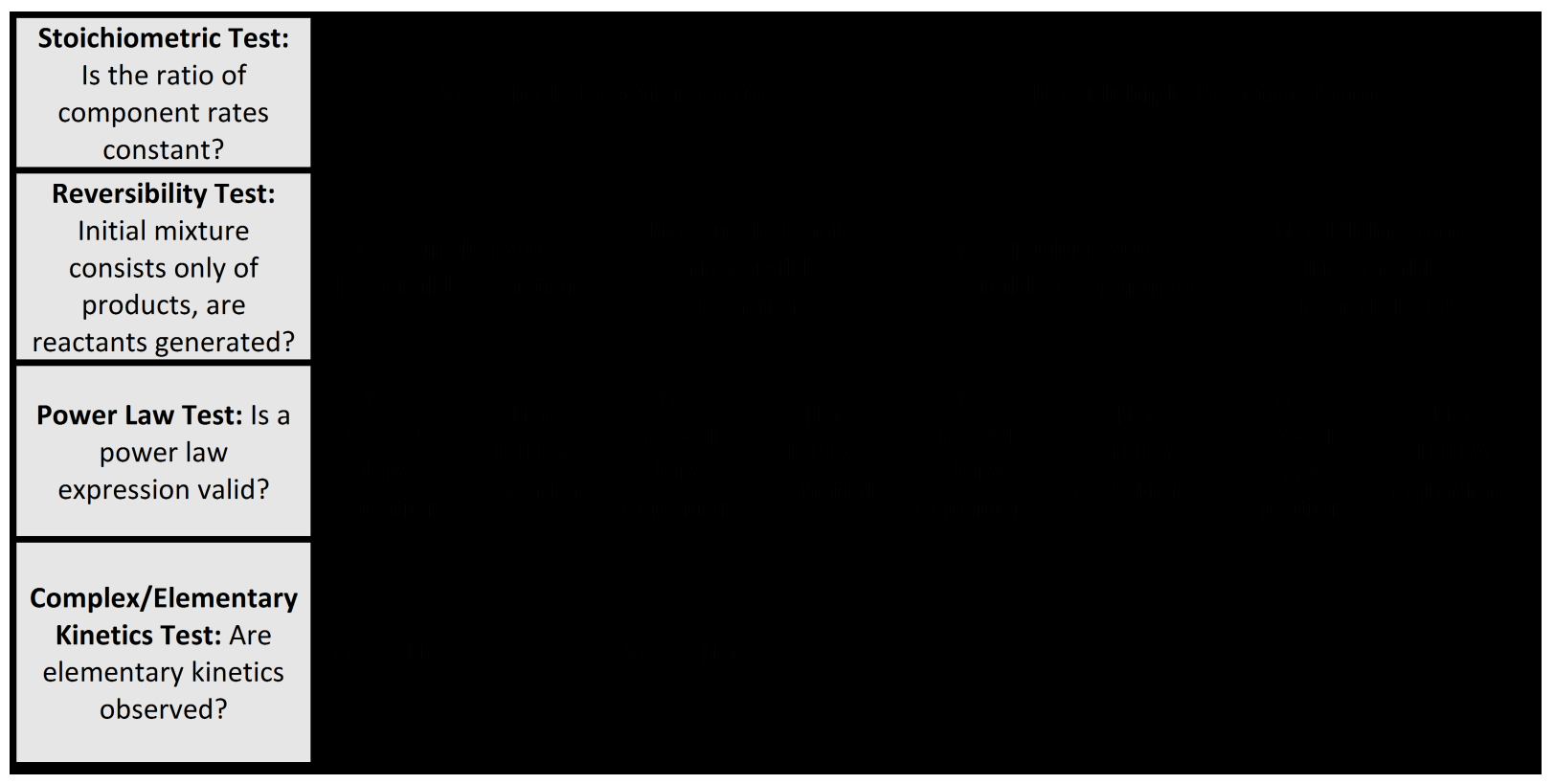

\subsubsection{Stoichiometric Test: Distinguishing Single and Multiple Route Reactions}

For distinguishing a single reaction route from a multiple route reaction, one must compare rates of changes of different components, reactants and products. From this comparison, one concludes whether the rates of different components are 'coherent' or not. If a ratio of these rates corresponds to the ratio of the stoichiometric coefficients of corresponding components, it is considered as a fingerprint of the single reaction.

For the single balanced overall reaction

in which $A$ and $B$ are reactants; $C$ and $D$ are products; $a, b, c$ and $d$ are stoichiometric coefficients; stoichiometric coefficients are considered to be negative for reactants and positive for products, respectively; $C_{A}, C_{B}, C_{C}$ and $C_{D}$ are concentrations of components $A, B, C$ and $D$, respectively.

Rates of changes of components $A, B, C$ and $D$ are: , respectively. Ratios of these rates must equal: 
Experimental kinetic data can then be tested regarding these relationships.

\section{Example}

In this example we will compare rates derived from batch reactor data using the differential method of kinetic analysis. Here the batch time, rather than space time, is the independent variable and the reaction rate follows from the time derivative. If concentrations, rather than conversions, are monitored then:, then the derivative can be approximated as a value, . In the oxidation reaction of ethylene to ethylene epoxide, $\mathrm{C}_{2} \mathrm{H}_{4}+\mathrm{O}_{3} \cdot \mathrm{C}_{2} \mathrm{H}_{4} \mathrm{O}+\mathrm{O}_{2}$, the concentration of reactants and products in Table 5 are measured as a function of time.

Table 5: Experimental concentration data for ethylene epoxidation.

\begin{tabular}{|l|l|l|l|l|}
\hline Time, $s$ & $\begin{array}{l}\text { Concentration of } \\
\mathrm{C}_{2} \mathrm{H}_{4}, \mathrm{~mol} \mathrm{~m}^{-3}\end{array}$ & $\begin{array}{l}\text { Concentration of } \\
\mathrm{O}_{3}, \mathrm{~mol} \mathrm{~m}^{-3}\end{array}$ & $\begin{array}{l}\text { Concentration of } \\
\mathrm{C}_{2} \mathrm{H}_{4} \mathrm{O}, \mathrm{mol} \mathrm{m} \mathrm{m}^{-3}\end{array}$ & $\begin{array}{l}\text { Concentration of } \\
\mathrm{O}_{2}, \mathrm{~mol} \mathrm{~m}^{-3}\end{array}$ \\
\hline 0.0 & $6.40 \times 10^{-5}$ & $3.20 \times 10^{-5}$ & 0.0 & 0.0 \\
\hline 10.0 & $5.62 \times 10^{-5}$ & $2.42 \times 10^{-5}$ & $0.78 \times 10^{-5}$ & $0.78 \times 10^{-5}$ \\
\hline 20.0 & $5.15 \times 10^{-5}$ & $1.95 \times 10^{-5}$ & $1.25 \times 10^{-5}$ & $1.25 \times 10^{-5}$ \\
\hline 30.0 & $4.83 \times 10^{-5}$ & $1.63 \times 10^{-5}$ & $1.57 \times 10^{-5}$ & $1.57 \times 10^{-5}$ \\
\hline 40.0 & $4.60 \times 10^{-5}$ & $1.40 \times 10^{-5}$ & $1.80 \times 10^{-5}$ & $1.80 \times 10^{-5}$ \\
\hline 50.0 & $4.43 \times 10^{-5}$ & $1.23 \times 10^{-5}$ & $1.97 \times 10^{-5}$ & $1.97 \times 10^{-5}$ \\
\hline 60.0 & $4.30 \times 10^{-5}$ & $1.10 \times 10^{-5}$ & $2.10 \times 10^{-5}$ & $2.10 \times 10^{-5}$ \\
\hline
\end{tabular}

For the reactant $\mathrm{O}_{3}$, within the time interval $[0.0 \mathrm{~s} ; 10.0 \mathrm{~s}]$, the average rate of change of this component is:

For the product, $\mathrm{O}_{2}$, within the same time interval $[0.0 ; 10.0]$, the average rate of change of this component is:

In a reaction, the concentration of reactants decrease and the concentration of products increase, thus the rate of reactant change is negative and the rate of product change is positive. Generally, for every $i$ th component, the average rate of its change within the temporal interval $\left[t_{2}, t_{7}\right]$ is:

For the reactant $\mathrm{O}_{3}$, over the time interval $[0.0 \mathrm{~s} ; 60.0 \mathrm{~s}$ ], the average rate of change is:

For the product, $\mathrm{O}_{2}$, in the same time interval, the rate is: 
The average rate value depends on the selective time interval, it is possible to determine the reaction rate even based on two initial concentration measurements. However, in this case accuracy will be limited. The initial reaction rate can be better estimated by extrapolating a line from several points early in the 'concentration-time' dependence.

If we compare the rates of $\mathrm{O}_{3}$ and $\mathrm{O}_{2}$ calculated over the $[0.0 \mathrm{~s} ; 10.0 \mathrm{~s}]$ time interval then:

The same relationship between the rates of reactant and product is found over the time interval [0.0 s; 60.0s]. Similar relationships are justified based on the data presented in Table 5 as well:

Finally, all Equations (98) - (99) can be presented as:

where $(-1),(-1) ;(+1),(+1)$ are stoichiometric coefficients of components $\mathrm{C}_{2} \mathrm{H}_{4}, \mathrm{O}_{3}, \mathrm{C}_{2} \mathrm{H}_{4} \mathrm{O}$ and $\mathrm{O}_{2}$, respectively; $\boldsymbol{r}$ is the reaction rate, a coherent characteristic of the single reaction. Finally, it can be concluded that the chemical transformation in this mixture occurs in accordance with the single reaction:

or, in a traditional way:

It must be stressed again that generally the reaction rate $\boldsymbol{r}$, is distinguished from the $\boldsymbol{R}_{\boldsymbol{i}}$, the net rate of change of the $\boldsymbol{i}^{\text {th }}$ component. In a general case, for a single reaction as described in (91), the following stoichiometric relationship is valid:
\end{abstract}


In contrast to the rates of change of components $R_{i}$, the reaction rate, $r$, is always positive. For example:

For single reaction $\mathrm{H}_{2}+\mathrm{I}_{2}=2 \mathrm{HI}$, Equation (103) is:

For single reaction $2 \mathrm{H}_{2}+\mathrm{O}_{2}=2 \mathrm{H}_{2} \mathrm{O}$, Equation (103) is:

If the stoichiometric relationships of type (103) are not justified, it is a clear fingerprint of multiple reactions. If the stoichiometric relationships are justified and a single reaction route is considered, the resulting integer stoichiometric coefficients (whole numbers) will be needed to determine whether the reaction is elementary or has a mechanism that of many elementary reactions (discussed in Section 4.2.4). In the elementary reaction, only such integer stoichiometric coefficients have a physico-chemical meaning: one, two, and three (rarely). Also, for any reaction, forward or reverse, the sum of these coefficients cannot exceed three. Therefore, if stoichiometric coefficients do not meet such requirements, the analyzed reaction is complex, and not elementary. However, even if the stoichiometric coefficients meet such requirements, it can be taken only as a necessary, but not a sufficient condition of the elementary reaction.

\subsubsection{Reversibility Test}

For a reversible reaction, the reaction rate is a combination of the rates of the forward and reverse reactions:

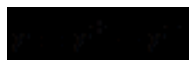

The state in which $d C / d t=0$, is called equilibrium. In this state and For a single reaction, first, we have to distinguish whether the overall reaction is reversible or irreversible. Typically, it is known before the experiment. However, it can be additionally proven based on the experimental data in which the initial composition will consist of only products (reactants are absent in the initial composition). If the transformation of products into reactants is not observed, the single reaction is irreversible. For example, in the reaction of methane combustion $\mathrm{CH}_{4}+2 \mathrm{O}_{2} \mathrm{CO}_{2}+2 \mathrm{H}_{2} \mathrm{O}$, reactants $\mathrm{CH}_{4}$ and $\mathrm{O}_{2}$ are not produced from the " $\mathrm{CO}_{2}$-water"- mixture. This reaction is a typical irreversible combustion process. Another example is the Water-Gas-Shift (WGS) Reaction, $\mathrm{CO}+\mathrm{H}_{2} \mathrm{O} \mathrm{CO}_{2}+\mathrm{H}_{2}$, a widely recognized reversible process which can go in both directions (both Water-Gas-Shift (WGS) Reaction and Reverse Water-Gas-Shift (rWGS) Reaction. Reactants $\mathrm{CO}$ and $\mathrm{H}_{2} \mathrm{O}$ can be produced from the mixture that contains only $\mathrm{CO}_{2}$ and $\mathrm{H}_{2}$. 


\subsubsection{Power Law Test}

The goal of this test is to verify a validity of the power law expression for expressing the reaction rate in terms of the reaction conditions. A distinction must be made for the case of irreversible and reversible reaction routes.

Irreversible Reaction Case

Assuming the reaction is irreversible, the following power law expression can be tested:

This expression is a power law expression where values of powers ? can be determined by varying the initial composition of the reaction mixture. For example, in the aqueous reaction between mercury (II) chloride and the oxalate ion: $2 \mathrm{HgCl}_{2}+\mathrm{C}_{2} \mathrm{O}_{4}{ }^{2-} \cdot 2 \mathrm{Cl}^{-}+2 \mathrm{CO}_{2}+\mathrm{Hg}_{2} \mathrm{Cl}_{2}$, the initial concentration and experimental rate data listed in Table 6 can be considered.

Table 6: Initial concentrations and rates $(1 \mathrm{M}=1 \mathrm{~mol} / \mathrm{L})$

\begin{tabular}{|l|l|l|l|}
\hline Experiment & $\begin{array}{l}\text { Initial concentration } \\
\text { of } \mathrm{HgCl}_{2,} \mathrm{M}\end{array}$ & $\begin{array}{l}\text { Initial concentration } \\
\text { of } \mathrm{C}_{2} \mathrm{O}_{4}^{-}, \mathrm{M}\end{array}$ & $\begin{array}{l}\text { Initial reaction rate, } \\
\mathrm{M} \mathrm{min}^{-1}\end{array}$ \\
\hline 1 & 0.105 & 0.15 & $r_{1}=1.8 \times 10^{-5}$ \\
\hline 2 & 0.105 & 0.30 & $r_{2}=7.1 \times 10^{-5}$ \\
\hline 3 & 0.052 & 0.30 & $r_{3}=3.5 \times 10^{-5}$ \\
\hline
\end{tabular}

In correspondence with Equation (105), ,

Consequently, (2) ${ }^{\alpha 2}=4$, and $\alpha_{2}=2$. The reaction rate is second order with respect to $\mathrm{C}_{2} \mathrm{O}_{4}$. Furthermore,

Consequently, (2) ${ }^{\alpha 1}=2$, and $\alpha_{1}=1$. The reaction rate is first order in $\mathrm{HgCl}_{2}$. For this reaction, the reaction rate can be presented by the equation: 
The kinetic coefficient (rate constant) can be calculated from Equation (108):

\section{Reversible Reaction Case}

For a reversible reaction, $r=r^{+}-r^{-}$, the Power Law Expression is written similar to Equation (105), where rates of forward and reverse reactions are expressed by power law dependences as follows:

where $C_{\mathrm{A} i}$ and $C_{\mathrm{B} i}$ are the concentrations of reactants and products $\left[\mathrm{mol} \mathrm{m}{ }^{-3}\right.$ ], the rate constants $k^{+}$and $k^{-}$are the coefficients that determine the reaction rates of the forward and the backward reaction at unitary values of reactant concentrations. The rate constants are governed by the Arrhenius dependences and increase exponentially with temperature:
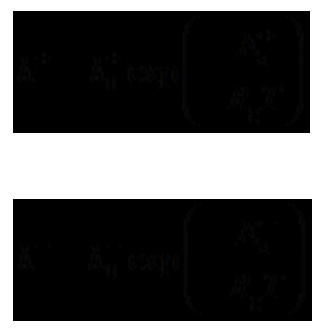

Here $\square$ and $\square$ are the pre-exponential factors, and are the activation energies of the forward and backward reactions, respectively; $R_{\mathrm{g}}$ is the universal gas constant, and $T$ is the absolute temperature. The ratio of the rate coefficients of the forward and reverse reaction determines the equilibrium constant $K_{\mathrm{eq}}=(/)$. The rate constant of the reverse reaction, , can be found based on 
known rate constant of the reverse reaction, , and equilibrium constant, $K_{\text {eq. }}$ In this case, the same kinetic expressions (108), (110) and (111) will be used, and the rate constant will be determined as:

Again, for power law dependences, there are no restrictions for values of powers, but they are typically equal to 0,1 or 2 and (rarely) 3. They can be fractional positive or negative as well. The obtained power law dependences are considered as semi-empirical expressions. However, simple power law dependences are invalid to describe experimental data in which values of powers are changed depending on the concentration and temperatures domain studied.

\subsubsection{Distinguishing Elementary Reactions}

\section{A Particular Case of the Power Law Kinetic Dependence}

A chemical reaction can be considered elementary only under the following conditions $n=1, n=2, n=3$ (rarely). A sum of all kinetic orders must be less than three. Hence, if for a reaction the kinetic order does not meet such requirements, this reaction is considered to be complex and is not an elementary reaction. The kinetic order can be found via concentration and conversion patterns.

\subsection{Non-Power Law Kinetic Dependences}

As mentioned previously, the simple power law dependences are invalid to describe experimental data in which values of powers are changed depending on the concentration and temperature domains studied. The typical non-power law kinetic dependence is the equation presented by Langmuir and Hinshelwood in the 1920s-1930s (LH-equation) for describing catalytic oxidation reactions, in particular $\mathrm{CO}$ and hydrogen oxidation over metals of the platinum group:

The Langmuir-Hinshelwood equation reflects complexity of the catalytic reaction: (i) a catalytic process involves competition between the components of the reaction mixture for sites on the catalyst surface, and (ii) very often adsorption and desorption rates are potentially high in comparison with other steps of chemical transformation on the catalyst surface.

Then, in the 1940s, Hougen and Watson proposed a similar semi-empirical rate equation, the HW equation, for a reversible complex catalytic reaction, considering it the basis of reactor design. For instance, for the dehydrogenation of cyclohexane this equation has the form: 
Hougen and Watson also presented the denominator in the form:

where are thermodynamic activities of reactants and products and $K_{\mathrm{A}}, K_{\mathrm{R}}, K_{\mathrm{I}}, \ldots$ are adsorption equilibrium coefficients of reactants and products.

Equations (115) and (116) are generally called Langmuir-Hinshelwood-Hougen-Watson (LHHW)equations indicating their similarity: they belong to the class of "numerator-divided-by denominator"kinetic equations. Within the HW approach, a rate-determining step is typically assumed. In many cases the adsorption/desorption steps are considered to be quasi-equilibrated. In many cases, a numerator and a denominator of the of the LHHW equation are defined as a 'kinetic term' and an 'adsorption term', respectively. The kinetic term relates to the kinetic dependence of the net equation, rather to say, it reflects some important features of the net kinetic dependences. In some typical cases, the numerator of the rate expression is considered to correspond to the net reaction. The adsorption term reflects complex transformations via intermediates (intermediates for the surface catalytic reaction).

For the LHHW-equations there are two physico-chemical requirements:

a) All coefficients of these equations must be positive,

b) If the numerator of the LHHW-equation has two terms which correspond to forward and reverse reactions respectively, with coefficients and, the relationship ( / ) $=K_{\text {eq,n }}$ must be fulfilled, where $K_{\text {eq,n }}$ is the equilibrium constant for the net reaction. It is an absolutely necessary thermodynamic requirement.

In the 1970s and 80s, Yablonsky et al. used the theory of graphs to formulate a 'recipe' with which writing of the steady-state rate equations is made very easy [35]. It was demonstrated for a so-called linear mechanism in which every elementary reaction, forward or reverse, includes only one molecule of the intermediate. For the case of a single-route complex reaction this recipe is very simple. First, the rate of the overall reaction is presented by assuming it is described by the mass action law. This expression will be the numerator of the steady-state rate equation. As for the denominator, it is written in accordance with the rules of the theory of graphs. It is a polynomial with respect to the concentrations of reactants and products. The denominator reflects the complexity of the chemical reaction; it characterizes the inhibiting effect of reactants and products on the reaction rate. Generally, all mentioned kinetic equations, including the Langmuir-Hinshelwood and Hougen-Watson equations, as well as equations constructed using graph theory are particular cases of a so-called 'kinetic polynomial' proposed by Lazman and Yablonsky see reference [5], Chapter 9. Different forms of the steady-state kinetic equation can be applied, for example: 
where $W$ is the denominator, and are combinations of kinetic coefficients, $Q$ is the quotient of the overall chemical reaction:

For the catalytic reaction of ammonia synthesis:

$$
\mathrm{N}_{2}+3 \mathrm{H}_{2} \rightleftarrows 2 \mathrm{NH}_{3}
$$

a special power law kinetic equation is the well-known Temkin-Pyzhev equation:

where $P_{\mathrm{N} 2}, P_{\mathrm{H} 2}$, and $P_{\mathrm{NH} 3}$ are partial pressures of nitrogen, hydrogen and ammonia, respectively; $f$ is the factor of the catalyst activity; and are kinetic coefficients for the forward and reverse reaction of ammonia synthesis, respectively; $(/)=K_{e q}$, where $K_{e q}$ is the equilibrium constant of reaction (123). Equation (124) can presented as a LHHW equation:

where $K_{3}$ is the apparent coefficient related to ammonia adsorption and $A$ is a parameter introduced to avoid non-physical cases; i.e., where at and.

\section{EXAMPLE}

Starting from the LHHW-equation, in our classification, it belongs to the class of the non-power-law kinetic dependences. Assuming the single reaction route for the catalytic reaction of methanol synthesis:

$$
\mathrm{CO}_{2}+3 \mathrm{H}_{2} \rightleftarrows \mathrm{CH}_{3} \mathrm{OH}+\mathrm{H}_{2} \mathrm{O}
$$


the kinetic law for the industrial catalyst $\mathrm{CuO} / \mathrm{ZnO} / \mathrm{Al}_{2} \mathrm{O}_{3}$ is expressed by the following dependence [36]:

where

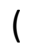

The equilibrium constant, $K_{e q}$, is determined as:

where the unit of $\mathrm{K}_{\mathrm{eq}}$ is $\mathrm{atm}^{-2}$. Since, $1 \mathrm{~atm}=1.01325 \mathrm{bar}$,

with the unit, bar $^{-2}$.

The kinetic coefficients were expressed as a product of a pre-exponential factor $(A)$ and an exponential term $\exp (-E / R T)$, where $\mathrm{E}$ is the activation energy:

$$
\begin{array}{rrrl}
k & A=0.00107 \text { mol }_{\left(g s \text { bar }^{-2}\right)} & (\mathrm{E} / \mathrm{R})=4414 \mathrm{~K} \\
\text { a1 } & A=0.499 \mathrm{bar}^{-0.5} & (\mathrm{E} / \mathrm{R})=2068 \mathrm{~K} \\
\text { a2 } & A=6.62 \times 10^{-11} \text { bar }^{-1} & (\mathrm{E} / \mathrm{R})=14928 \mathrm{~K} \\
\text { a3 } & A=3453.38 \text { (dimensionless) } & (\mathrm{E} / \mathrm{R})=0
\end{array}
$$


The kinetic term can be presented as a difference of two terms, 'forward' and 'reverse' ones:

Obviously, this form of the kinetic term relates to the kinetic dependences of the net reaction $\mathrm{CO}_{2}+$ $3 \mathrm{H}_{2} \backsim \mathrm{CH}_{3} \mathrm{OH}+\mathrm{H}_{2} \mathrm{O}$. The LHHW equation (127) is significantly simplified in the domain of small concentrations of hydrogen and water in which terms $a_{7}\left(P_{\mathrm{H}_{2}}\right)^{0.5}, a_{2} P_{\mathrm{H} 2 \mathrm{O}}$ and $a_{3}\left(P_{\mathrm{H} 2 \mathrm{O}} / P_{\mathrm{H} 2}\right)$ are small in comparison with 1 , and the adsorption term is degenerated to 1 . In this case this equation is applied to the description of kinetic dependences:

The next level of simplification occurs in the temperature domain in which the reversibility of the reaction can be neglected; then the following expression is used:

\subsection{Time-Dependent Features and Fingerprints}

In this section we will for the sake of simplicity take the case of a batch reactor. It is sufficient to substitute the batch time $t$ by the space time $\tau$ to go from batch to ideal continuous plug flow reactors.

\subsubsection{Concentration Time Dependence and Reaction Half-Life}

Assuming the single reaction $\mathrm{A} \cdot \mathrm{B}$ and the rate of the component change $R=k C_{a}{ }^{n}$, where $k$ is the kinetic rate coefficient and $n$ is an apparent reaction order, one can distinguish the apparent order $n$ based on the concentration change in time. A useful concept is the 'half-life', $t_{1 / 2}$, i.e., time during which a half of the reactant concentration is consumed, $C_{A}=1 / 2 C_{A O}$.

i) The first order reaction, $r=k C_{A}$

A linear dependence, $\ln \left(C_{a}\right)$ versus $t$, is observed:

where the slope is the kinetic coefficient $(-k)$. The half-life is determined as follows: $t_{1 / 2}=0.693 / k$. It does not depend on the initial concentration.

$$
k=0.693 / t_{1 / 2}
$$


ii) The second order reaction, $r=k C_{A}^{2}$

A linear dependence, $\ln \left(1 / C_{a}\right)$ versus $t$, is observed:

where the slope is the kinetic coefficient $(+k)$. The half-life is determined as follows: $t_{1 / 2}=1 /\left(k C_{A 0}\right)$. It is inversely proportional to the initial concentration.

iii) The zero-order reaction, $r=k$

A linear dependence, $C_{a}$ versus $t$, is observed:

where the slope is the kinetic coefficient $(-k)$. The half-life is determined as follows: $t_{1 / 2}=C_{A 0} /(2 k)$. It is proportional to the initial concentration.

iv) The $n$-order reaction, $r=k C_{A}{ }^{n}$

A linear dependence, $\left(1 / C_{A}\right)^{(n-1)}$ versus $t$, is observed:

v) The first-order reversible reaction $A$ ? $B$

With the dependences and, where and are rates of forward and backward rates, respectively; and are the rate coefficients of forward and backwards rates, respectively; $C_{A}$ and $C_{B}$ are concentrations of substances $A$ and $B$, respectively. In the batch reactor, $C_{A}+C_{B}=1$. A fingerprint of the reversible reaction is that the final reactant conversion is not complete $\left(C_{A \text { r final }}=C_{A \text { req }}\right)$, which is not equal 0$)$. In this case, a linear dependence, $\left(C_{A O}-C_{A, e q}\right)$ versus $t$, is observed. The concentration temporal dependence will assume: 
where $C_{A_{r e q}}=1 /\left(1+K_{e q}\right), C_{\text {breq }}=K_{e q} /\left(1+K_{e q}\right), K_{\text {eq }}$ is an equilibrium constant, $K_{\text {eq }}=(/)$. In this case, $K_{\text {eq }}=C_{B_{r} \text { eq }} / C_{A_{\text {req }}}$ and $C_{B_{\text {req }}}=1-C_{A_{\text {req }}}$. $K_{\text {eq }}=\exp (-\Delta G / R T)$, where $\Delta G$ is the standard Gibbs energy change of reactions obtained from literature, $R$ is gas constant and $T$ is the absolute temperature. The sum of kinetic coefficients ( and ), and then, individual coefficients and, can be found via:

and $K_{e q}=(/)$.

\subsubsection{Quasi-Equilibrium and Quasi-Steady-State}

In the steady-state kinetic reactor the temporal change of a component is not observed, , while in the non-steady-state reactor concentration of components may change in time, . Equilibrium is a particular case of steady-state, it is also the final state achieved from non-steady-state behavior of a closed chemical system, e.g., a batch reactor. The principle of detailed equilibrium demonstrates that at the point of equilibrium, not only do the concentrations remain unchanged in time, , but also the rate of each forward rate is balance by that of its associated reverse counterpart. For the triangular chemical mechanism:

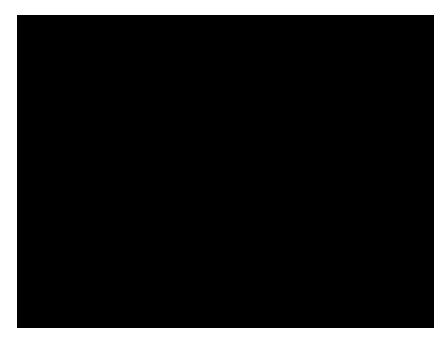

at the point of detailed equilibrium:

where are the corresponding forward and reverse rates, are the concentrations of substances $\mathrm{A}, \mathrm{B}$ and $\mathrm{C}$, respectively, are kinetic coefficients of corresponding forward and reverse reactions, respectively. From balance equations ( it follows and where are equilibrium constants of the corresponding reactions. 
The quasi-equilibrium regime is a special non-steady-state regime at which the ratio of some concentration remains approximately constant during a certain temporal domain. This ratio is equal to the equilibrium constant of the 'fast' reaction. The simplest example of a quasi-equilibrium regime is:

where $>$. Figure 14 illustrates the domain of quasi-equilibrium where .

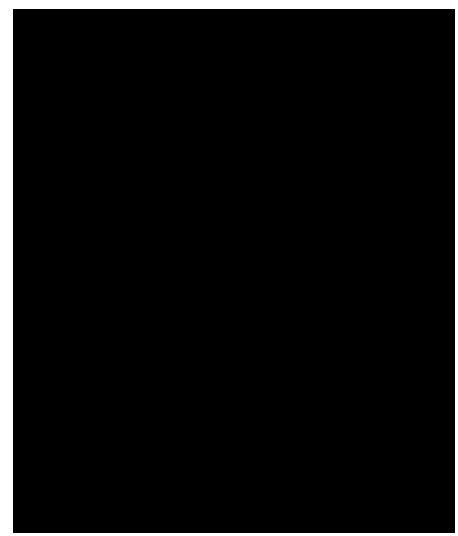

Figure 14: Illustration of a quasi-equilibrium regime.

The quasi-steady-state regime is a special non-steady-state regime where the ratio of some concentrations remains approximately constant during a certain temporal domain. It results where a small kinetic parameter is divided by a fast kinetic parameter. The simplest example of the quasisteady-state regime is the following:

where if >> then one would observe trends as illustrated in Figure 15; in this case .

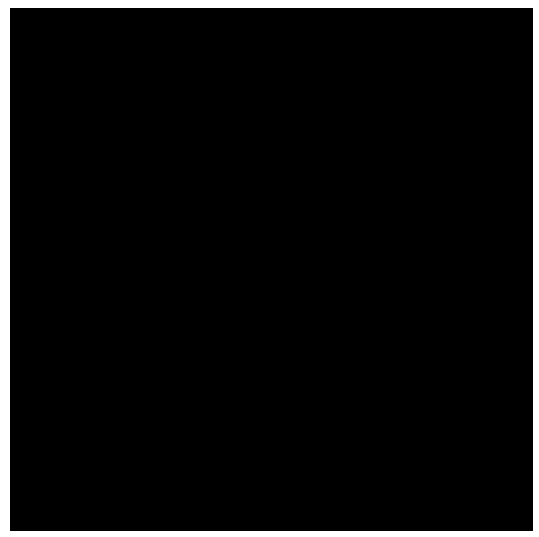

Figure 15: Illustration of a quasi-steady state regime.

Two origins of quasi-steady-state regimes for different substances can be distinguished:

a) Where a significant difference exists between kinetic coefficients of some steps,

b) Where a significant difference exists between relative numbers, e.g., the number of gas molecules is much larger than then number of surface active sites. 
Quasi-equilibrium and quasi-steady-state regimes can be distinguished as follows. Under quasiequilibrium conditions, some concentrations are comparable in magnitude and their ratio is determined by the equilibrium constant of the corresponding reactions; i.e. in the proceeding example $C_{A}$ and $C_{B}$ are comparable. Under quasi-steady-state conditions, some concentrations are small. At these conditions, the ratio of a small concentration is divided by a much larger concentration and hence the ratio remains approximately constant, e.g., in the previous example, $C_{B} \ll C_{A}, C_{C}$ and.

\subsubsection{Conversion Time-Dependence}

Conversion may be calculated as $X_{A}=\left(C_{A O}-C_{A}\right) / C_{A O}$, where $C_{A O}$ is an initial concentration for a batch reaction and $C_{A}$ is the concentration in the reactor at time, $t$. In Figure 16, temporal kinetic dependences observed in a batch reactor for the reaction $A \cdot B$ is shown for different values of kinetic order, $n$.

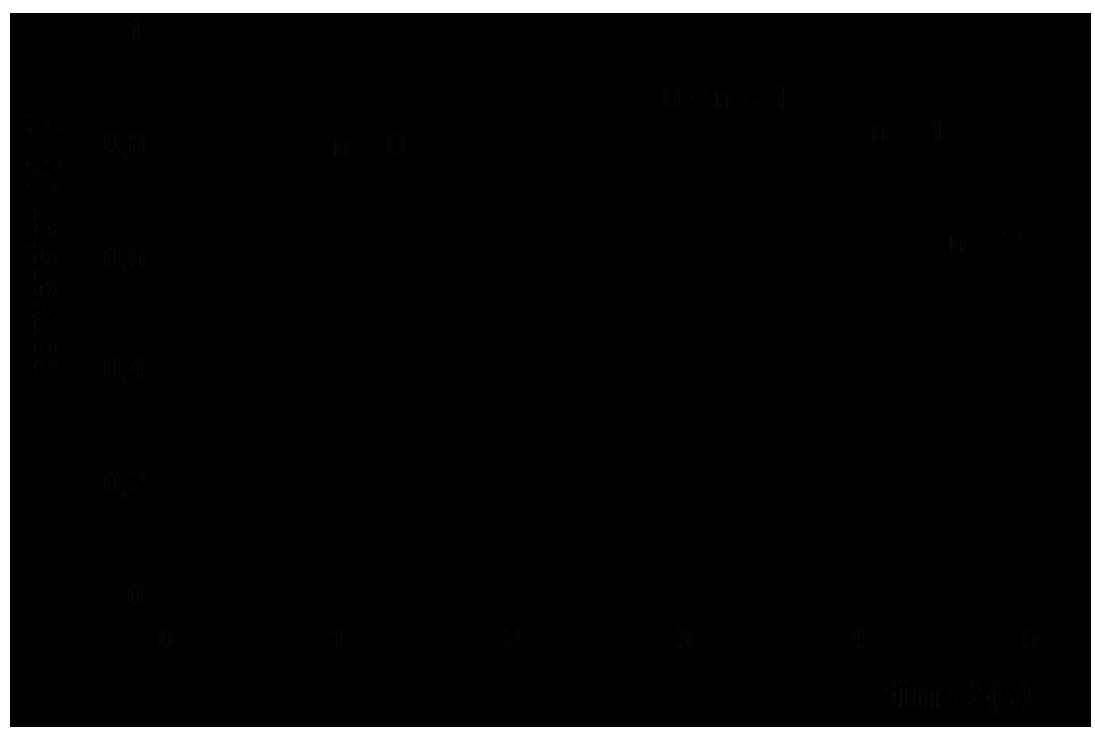

Figure 16: Temporal kinetic dependences in a batch reactor for the reaction $A$ a $B$ with, for different values of $n$. Reproduced from [5].

In terms of conversion, for the first order reaction, , the expression for conversion is and at small times, and $k$ is estimated as .

For the second order reaction, , the expression for conversion is and at small times, and $k$ is estimated as

For the zero order reaction, , the expression for conversion is and $k$ is estimated as

For the fractional $n$-order reaction, with $0<\mathrm{n}<1$, the expression for conversion is . 


\section{Conclusions}

Chemical kinetics is a cross-roads of pure and applied sciences, including physical chemistry and physics, catalysis and chemical engineering in addition to mathematics. The following advancements have been crucial for chemical kinetics in the past 40 years:

1) The development of new experimental and analytical techniques that enable monitoring the chemical composition of a multicomponent reaction mixture under both steady-state and nonsteady-state conditions,

2) The development of a battery of new physical methods which enable precise characterization of both the catalyst structure and surface intermediates, ideally under operando conditions,

3) Finally, the design of new theoretical (mathematical and computational) tools and approaches for decoding the complexity of chemical reactions.

The future of chemical kinetics lies in the efficient integration of these important developments. 


\section{References}

[1] M. Boudart, Turnover rates in heterogeneous catalysis, Chemical reviews, 95 (1995) 661-666.

[2] M. Boudart, H. Brenner, Kinetics of Chemical Processes: Butterworth-Heinemann Series in Chemical Engineering, Elsevier Science, 2014. [3] M. Boudart, G. Djéga-Mariadassou, Kinetics of heterogeneous catalytic reactions, Princeton University Press Princeton, 1984. [4] M.A. Vannice, W.H. Joyce, Kinetics of catalytic reactions, Springer, 2005. [5] G.B. Marin, G.S. Yablonsky, D. Constales, Kinetics of chemical reactions: Decoding complexity, Wiley$\mathrm{VCH}$,

[6] D. Murzin, T. Salmi, Catalytic kinetics, Elsevier Amsterdam, 2005. [7] C.G. Hill, An Introduction to Chemical Engineering Kinetics and Reactor Design, Wiley, 1977. [8] R.J. Berger, F. Kapteijn, J.A. Moulijn, G.B. Marin, J. De Wilde, M. Olea, D. Chen, A. Holmen, L. Lietti, E. Tronconi, Dynamic methods for catalytic kinetics, Applied Catalysis A: General, 342 (2008) 3-28. [9] R.J. Berger, E.H. Stitt, L. Chewter, J. Verstraete, G.B. Marin, J. Hoorn, The Eurokin consortium: origin, topics and aims, Green Processing and Synthesis, 2 (2013) 67-69. [10] C.H. Bartholomew, R.J. Farrauto, Fundamentals of Industrial Catalytic Processes, Second Edition ed., John Wiley \& Sons, Inc., Hoboken, New Jersey, 2006. [11] R.J. Wijngaarden, A. Kronberg, K.R. Westerterp, Industrial Catalysis: Optimizing Catalysts and Processes, Wiley-VCH Gerlag 1998. [12] J.M. Berty, Experiments in catalytic reaction engineering, in, Elsevier, Amsterdam; New York, 1999. [13] E.G.P.Z. Christoffel, Laboratory studies of heterogeneous catalytic processes, in, Elsevier, Amsterdam; New $\quad$ York, 1989. [14] D. Murzin, Engineering Catalysis, in, De Gruyter, Berlin, 2013. [15] J. Berty, Testing commercial catalysts in recycle reactors, Catalysis Reviews Science and Engineering, 20 (1979) 75-96.

[16] J.M. Berty, Applied industrial catalysis, in: B. Leach (Ed.), Access Online via Elsevier, 1983, pp. 41-67. [17] J. Carberry, Designing laboratory catalytic reactors, Industrial \& Engineering Chemistry, 56 (1964) 39-46.

[18] G.F. Froment, K.B. Bischoff, J. De Wilde, Chemical reactor analysis and design, Wiley New York, 1990.

[19] L. Forni, Laboratory reactors, Catalysis today, 34 (1997) 353-367. [20] J.R. Ebner, J.T. Gleaves, Method and apparatus for carrying out catalyzed chemical reactions and for studying catalysts, 1986. [21] K. Morgan, N. Maguire, R. Fushimi, J. Gleaves, A. Goguet, M. Harold, E. Kondratenko, U. Menon, Y. Schuurman, G. Yablonsky, Forty years of temporal analysis of products, Catalysis Science \& Technology, 7 (2017) 2416-2439.

[22] S.O. Shekhtman, G.S. Yablonsky, S. Chen, J.T. Gleaves, Thin-zone TAP-reactor - theory and $\begin{array}{llllll}\text { application, } & \text { Chem. } & \text { Eng. } & \text { Sci., } & 54 & \text { (1999) }\end{array}$ [23] E.A. Redekop, G.S. Yablonsky, D. Constales, P.A. Ramachandran, C. Pherigo, J.T. Gleaves, The YProcedure methodology for the interpretation of transient kinetic data: Analysis of irreversible adsorption, Chem. $\quad$ Eng. $\quad$ Sci., $66 \quad$ (2011) 6441-6452. [24] G.S. Yablonsky, D. Constales, S.O. Shekhtman, J.T. Gleaves, The Y-procedure: How to extract the chemical transformation rate from reaction-diffusion data with no assumption on the kinetic model, $\begin{array}{lllll}\text { Chemical } & \text { Engineering } & \text { Science, } & 62 & \text { (2007) 6754-6767. }\end{array}$ [25] S.O. Shekhtman, G.S. Yablonsky, J.T. Gleaves, R. Fushimi, "State defining" experiment in chemical kinetics-primary characterization of catalyst activity in a TAP experiment, Chemical engineering science, 
[26] D. Constales, G.S. Yablonsky, L. Wang, W. Diao, V.V. Galvita, R. Fushimi, Precise non-steady-state characterization of solid active materials with no preliminary mechanistic assumptions, Catalysis Today, (2017).

[27] P. Phanawadee, S.O. Shekhtman, C. Jarungmanorom, G.S. Yablonsky, J.T. Gleaves, Uniformity in a thin-zone multi-pulse TAP experiment: numerical analysis, Chem. Eng. Sci., 58 (2003) 2215-2227.

[28] D.E. Mears, Diagnostic criteria for heat transport limitations in fixed bed reactors, Journal of Catalysis, 20

127-131.

[29] S.O. Shekhtman, G.S. Yablonsky, Thin-Zone TAP Reactor versus Differential PFR: Analysis of Concentration Nonuniformity for Gas-Solid Systems, Ind. Eng. Chem. Res., 44 (2005) 6518-6522. [30] Y. Wang, M.R. Kunz, S. Siebers, H. Rollins, J. Gleaves, G. Yablonsky, R. Fushimi, Transient Kinetic Experiments within the High Conversion Domain: The Case of Ammonia Decomposition, Catalysts, 9 (2019) 104.

[31] T.H. Chilton, A.P. Colburn, Mass transfer (absorption) coefficients prediction from data on heat transfer and fluid friction, Industrial \& engineering chemistry, 26 (1934) 1183-1187. [32] A.S. Gupta, G. Thodos, Mass and heat transfer in the flow of fluids through fixed and fluidized beds of spherical particles, Al Ch. E.(Am. Inst. Chem. Engrs.) J., 8 (1962). [33] J.D. Acetis, G. Thodos, Mass and heat transfer in flow of gases through spherical packings, Industrial $\begin{array}{lllll}\text { \& } & \text { Engineering } & \text { Chemistry, } & 52 & \text { (1960) }\end{array}$ [34] L. Doraiswamy, M. Sharma, Heterogeneous reactions: Analysis examples and reactor design. Vol. 1: Gas solid and solid-solid reactions, John Wiley and Sons, New York, (1984). [35] G.v. Yablonskii, V. Bykov, V. Elokhin, A. Gorban, Kinetic models of catalytic reactions, Elsevier, 1991. [36] K.V. Bussche, G. Froment, A steady-state kinetic model for methanol synthesis and the water gas shift reaction on a commercial cu/ZnO/al2o3catalyst, Journal of Catalysis, 161 (1996) 1-10. 